\title{
Custom-made macroporous bioceramic implants based on triply-periodic minimal surfaces for bone defects in load-bearing sites
}

Authors: Baptiste CHARBONNIER ${ }^{a}$, Mathieu MANASSERO ${ }^{b, c}$, Marianne BOURGUIGNON ${ }^{b, c}$, Adeline DECAMBRON ${ }^{b, c}$, Hanane ELHAFCI $^{b, c}$, Claire MORIN ${ }^{a}$, Diego LEON ${ }^{a}$, Morad BENSIDOUM ${ }^{b, c}$, Simon CORSIA ${ }^{b, c}$, Hervé PETITE ${ }^{b, c}$, David MARCHAT ${ }^{a}$, Esther POTIER ${ }^{b, c^{*}}$

\author{
Affiliations: \\ a. Mines Saint-Etienne, Univ Lyon, Univ Jean Monnet, INSERM, U 1059 Sainbiose, Centre CIS, Saint-Etienne, France \\ b. Université de Paris, CNRS, INSERM, B3OA, Paris, France \\ c. Ecole Nationale Vétérinaire d'Alfort, B3OA, Maisons-Alfort, France
}

Key words: Bone repair; Additive manufacturing; Calcium phosphates; Triply-periodic minimal surface (TPMS); Implant

To cite this article: Baptiste Charbonnier, Mathieu Manassero, Marianne Bourguignon, Adeline Decambron, Hanane El-Hafci, Claire Morin, Diego Leon, Morad Bensidoum, Simon Corsia, Hervé Petite, David Marchat, Esther Potier. Custom-made macroporous bioceramic implants based on triply-periodic minimal surfaces for bone defects in load-bearing sites. Acta Biomaterialia, Volume 109, 2020, Pages 254-266, https://doi.org/10.1016/j.actbio.2020.03.016.

Document Version: Accepted manuscript including changes made at the peer-review stage.

\begin{abstract}
The architectural features of synthetic bone grafts are key parameters for regulating cell functions and tissue formation for the successful repair of bone defects. In this regard, macroporous structures based on triplyperiodic minimal surfaces (TPMS) are considered to have untapped potential. In the present study, custom-made implants based on a gyroid structure, with (GPRC) and without (GP) a cortical-like reinforcement, were specifically designed to fit an intended bone defect in rat femurs. Sintered hydroxyapatite implants were produced using a dedicated additive manufacturing technology and their morphological, physico-chemical and mechanical features were characterized. The implants' integrity and ability to support bone ingrowth were assessed after 4, 6 and 8 weeks of implantation in a 3-mm-long, femoral defect in Lewis rats. GP and GPRC implants were manufactured with comparable macro- to nano-architectures. Cortical-like reinforcement significantly improved implant effective stiffness and resistance to fracture after implantation. This cortical-like reinforcement also concentrated new bone formation in the core of the GPRC implants, without affecting newly formed bone quantity or maturity. This study showed, for the first time, that custom-made TPMS-based bioceramic implants could be produced and successfully implanted in load-bearing sites. Adding a cortical-like reinforcement (GPRC implants) was a relevant solution to improve implant mechanical resistance, and changed osteogenic mechanism compared to the GP implants.
\end{abstract}

Graphical abstract

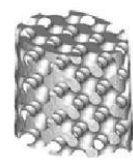

GP

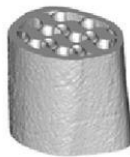

GPRC

$448 \pm 60 \mathrm{MPa}$

$654 \pm 85 \mathrm{MPa}$

Improved effective stiffness

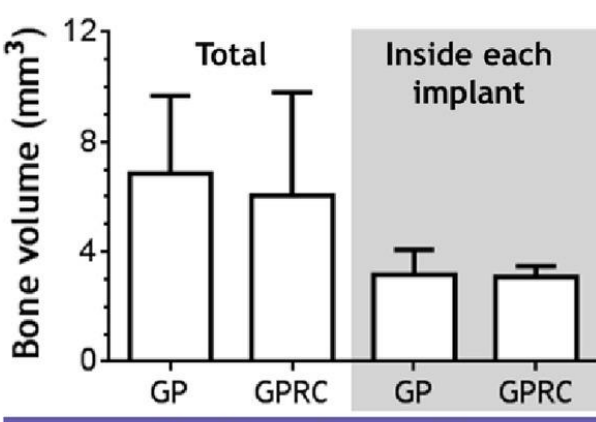

Similar volume of newly-formed bone 


\section{Introduction}

Despite the high regenerative capacity of human bone, large bone defects cannot heal spontaneously and require dedicated reconstruction surgical techniques. Each year, more than 2.2 million bone graft-related surgeries are performed worldwide [1] to augment bone repair after fracture, tumor resections and orthopedic revision surgeries, making bone the second most widely transplanted tissue after blood. Although autologous bone grafts are the "gold standard" for bone graft material, the considerable morbidity (8.5-20\% of reported complications [2]) associated with the donor site and the limited tissue quantity that can be harvested constitute serious limitations for this therapeutic modality. Allogenic bone grafts, obtained from either cadaver or living donors, are readily available and may be suitable alternatives to autologous bone grafts. Processing methods, however, are burdensome and costly; most importantly, risks of viral or bacterial infection remain serious clinical concerns [2]. To overcome these limitations, synthetic bone grafts, or alloplasts, have been proposed as bone substitutes or bone filling materials. The most popular alloplasts used in clinical practice are calcium phosphate $(\mathrm{CaP})$ bioceramics, which include hydroxyapatite (HA) and tricalcium phosphate, owing to their biocompatibility, safety, and osteoconductive properties.

An ideal alloplast should not only provide a temporary structural support in clinical bone treatments, but should also promote new bone tissue and blood vessel ingrowth. Formation of new tissues within the implant porous network (e.g., bone tissue growth [3], blood vessel guidance [4,5]), implant intrinsic features (e.g., mechanical properties [6,7], internal mass transport [8,9], resorption rate $[3,10])$, as well as cellular proliferation and differentiation $[11,12]$ can be modulated by the nano- to macroscale implant architecture. Additive manufacturing (AM) technologies currently enable production of custom-made implants, with well-controlled and diverse multiscale architectures, and with specific shapes designed to match the bone defect.

Macro-architectures based on triply-periodic minimal surfaces (TPMS) which include gyroids, diamonds, and primitives [13,14] have great potential for bone repair due to their open, regular, interconnected porous networks that facilitate mass transport, cell invasion, and tissue formation. These material architectures are also considered "biomorphic" because their mean curvature resembles the surface curvature characteristics of trabecular bone [15]. In vitro studies using non-ceramic materials have confirmed higher permeability of 3D implants and a better mesenchymal stromal cell infiltration in a gyroid network compared to a structure with a random-pore architecture of similar porosity and pore size [8]. Easily 3D-printed with either polymeric [7,8,16], metallic [17], [18], [19], [20], or composite [21], [22], [23] materials, TPMS structures are difficult to produce with $\mathrm{CaP}$ [24,25], due to the inability of most present-day AM to process bioceramics.

Using a precise approach based on the impregnation of wax molds, the authors were able to produce for the first time such TPMS structures in sintered HA [25]. Using these open, interconnected structures, the objective of the present study was to investigate the osteoconductive potential of TPMS-based HA implants (implants with gyroid porosity; GP) in large bone defects in a load-bearing, femoral site in rats. Because CaP bioceramics have well-established bioconductive and biointegrative capacity but poor fracture toughness [26], the ability of a cortical-like outer shell (implants with gyroid porosity reinforced by a cortical-like outer shell; GPRC) to improve the mechanical strength of the TPMS-based HA implants and its impact on bone ingrowth were evaluated.

\section{Materials and Methods}

Custom-made macroporous implants based on a TPMS gyroid structure, with (GPRC implants) and without (GP implants) a cortical-like outer shell, were produced in HA to fit a 3-mm-long, segmental, femoral defect in rats. Produced implants were characterized for their physico-chemical characteristics using Xray diffractometer (XRD) and Fourier transform infrared (FITR) spectroscopy, for their morphometric features using X-ray microcomputed tomography (micro-CT) and scanning electron microscopy (SEM), and for their mechanical properties using compression tests. The ability of GP and GPRC implants to support bone ingrowth in vivo was then evaluated by implanting them in a 3-mm-long, segmental, femoral defect in Lewis rats. Implant integrity and bone formation were qualitatively evaluated using X-ray micro-CT after 4, 6 and 8 weeks of implantation. Bone formation was quantified using $\mathrm{X}$-ray micro-CT, histomorphometry and SEM-energy-dispersive X-ray (EDX) spectroscopy after 8 weeks of implantation.

\section{Hydroxyapatite powder synthesis}

HA powder $\left(\mathrm{Ca}_{10}\left(\mathrm{PO}_{4}\right)_{6}(\mathrm{OH})_{2}\right)$ was synthesized using a standard aqueous precipitation method as previously described [27]. Briefly, a di-ammonium hydrogen phosphate solution $\left(\left(\mathrm{NH}_{4}\right)_{2} \mathrm{HPO}_{4}, 99 \%\right.$; Merck, Germany) was poured into a calcium nitrate solution $\left(\mathrm{Ca}\left(\mathrm{NO}_{3}\right)_{2} \bullet 4 \mathrm{H}_{2} \mathrm{O}, 99 \%\right.$; Merck, Germany) at 100 $\mathrm{mL} / \mathrm{min}$. The reaction was performed at $\mathrm{pH} 8.0$ and at a temperature of $65^{\circ} \mathrm{C}$ under argon flow. After a maturation time of $20 \pm 2 \mathrm{~h}$, the resulting HA suspension was filtered and the precipitate was heat-treated at $400^{\circ} \mathrm{C}$ under air for $2 \mathrm{~h}$ and crushed to reach particle sizes in the 1 to $10 \mu \mathrm{m}$ range (AFG100; Hosokawa Alpine, Germany). Finally, the synthetized HA powder was heat-treated at $1020^{\circ} \mathrm{C}$ for $2 \mathrm{~h}$ (LH40/13; Nabertherm, Germany) to obtain a specific surface area of $4.0 \pm 0.1 \mathrm{~m}^{2} / \mathrm{g}$ [25], which was determined on the outgassed powder $\left(200^{\circ} \mathrm{C}\right.$ for $\left.8 \mathrm{~h}\right)$ using N2 adsorption isotherms (Brunauer-Emmett-Teller 5 points method, ASAP 2010; Micromeritics, Germany).

\section{Manufacturing of hydroxyapatite implants}

HA implants were produced according to the AM-based process detailed and optimized by Charbonnier et al. [25,28]. Briefly, wax molds, negative of the intended implant structure, were build layer-by-layer with each layer thickness of $25 \mu \mathrm{m}$ (Fig. $\underline{1(A))}$ using a 3D printer (3Z Studio, Solid-scape; Multistation, France). These wax molds were then impregnated with a HA slurry. The HA slurry was prepared by blending $73.6 \%$ (w/w) HA powder, $26.2 \%(\mathrm{w} / \mathrm{w})$ pure water and $0.2 \%(\mathrm{w} / \mathrm{w})$ dispersing agent (Darvan C-N; R. T. Vanderbilt Company Inc., USA)), with stirring (at $200 \mathrm{rpm}$ ) in a zirconia jar containing 10- and 5-mm-diameter zirconia balls (PM400; Retsch, Germany) for 15 min. Duramax B1000 (Rohmand Haas, France) was finally added to the HA slurry prior to impregnation at a concentration of $3.7 \%$ (w/w of slurry).

After drying at room temperature overnight, the "green bodies" (i.e., agglomerates of particles) were cleaned then heat-treated in a debinding furnace (Carbolite, UK) at $500^{\circ} \mathrm{C}$ (to eliminate the wax mold and the organic adjuvants). Consolidation of the ceramic was achieved by sintering in air at $1200^{\circ} \mathrm{C}$ with a heating rate of 
$4^{\circ} \mathrm{C} / \min$ (LH40/13; Nabertherm, Germany) for $2 \mathrm{~h}$. Manufactured implants were sterilized at $121^{\circ} \mathrm{C}$ for $20 \mathrm{~min}$.

\section{Design of hydroxyapatite implants}

Implants were designed to match the geometry of a standardized, rat, 3-mm-long, segmental, femoral defect, using computer-aided-design (CAD) software (Fig. 1(B)). Lewis rats (female, 12-15 weeks old; Janvier Labs, France) were anesthetized and maintained under anesthesia using 2-3\% isoflurane (CS Pharmaceutiques, France) in $50 \%$ oxygen $/ 50 \%$ air. Rat femurs were then imaged with a non-invasive in vivo $\mathrm{X}$-ray micro-CT (Skyscan 1176; Bruker, Belgium) at the following settings: pixel size $=35.4 \mu \mathrm{m}$; source voltage $=65 \mathrm{kV}$; source current $=373 \mu \mathrm{A}$; filter $=$ Aluminum $1 \mathrm{~mm}$; rotation step $=0.7^{\circ}$; and exposure time $=$ $58 \mathrm{~ms}$. After X-ray micro-CT data reconstruction (CTAn; Brucker, Belgium), 3D models of the femurs were generated using ScanIP (Simpleware, UK), and a 3-mm-long volume in the rat femoral shaft was defined as the "basic volume" for the generation of the GP and GPRC implants. For the GP implants, a gyroid porous (60\% porosity) structure, where a sphere of $430-\mu$ m-diameter could move freely (equivalent gyroid unit of $810 \mu \mathrm{m}$ ) (Supplementary Fig. 1), was placed into this 3D volume (ScanIP) (Fig. 1(B)). For the GPRC implants, gyroid architecture similar to the GP implant was used but a cortical-like outer shell of 180- to 230- $\mu$ m-thick was left intact (Fig. 1(B)); this design resulted in a total porosity of $43 \%$.

As previously explained (Section "Manufacturing of hydroxyapatite implants"), molds were designed as the negative of the intended GP and GPRC implant architectures using ScanIP software and with an homogeneous expansion of $14 \%$ for both structures in order to counterbalance the uniform shrinkage of the HA green bodies occurring during their sintering [25].

\section{Hydroxyapatite powder and implant characterization}

The crystalline phases of the samples of interest to the present study were analyzed using a D8 Advance $\theta / \theta$ XRD (Bruker, Belgium) equipped with a Lynx-Eye Position Sensitive Detector (aperture angle $2.946^{\circ}$ ), using $\mathrm{CuK} \alpha$ radiation, and operating at 40 $\mathrm{kV}$ and $20 \mathrm{~mA}$. XRD patterns were collected over the $2 \theta$ range of $10-120^{\circ}$ at a step size of $0.01^{\circ}$ and counting time of $0.2 \mathrm{~s}$ per step. The crystalline phases were identified in comparison with reference patterns from the ICDD-PDF database (International Centre for Diffraction Data - Powder Diffraction Files). HA powder and grinded implants were also analyzed by FTIR spectroscopy using a VERTEX 70 spectrometer (Bruker Optics, France), equipped of a monolithic diamond ATR crystal (Quest ATR diamond; Specac, USA). Spectra were recorded from 4000 to $400 \mathrm{~cm}^{-1}$ at a resolution of $2 \mathrm{~cm}^{-1}$ and obtained by signal averaging of 64 successive scans.

Morphometric analyzes of the produced implants were carried out at various scales. Each implant was first imaged using a X-ray micro-CT system (Nanotom S, USA) with a voltage of $80 \mathrm{kV}$ (tungsten target), an integration time of $750 \mathrm{~ms}$, and a $3.5 \mathrm{~mm}$ voxel resolution. For the reconstruction of the volume data, a proprietary implementation method based on the Feldkamps cone beam-reconstruction algorithm was used. VG Studio software (Volume Graphics, Germany) was used for the 3D visualization of the volume data and the dataset export in .DICOM format for image analysis. For both implant architectures of interest in the present study, 3D models were generated in ScanIP using thresholding operations, and then, exported in a printable .stl format. After manual, gross superimposition of produced implant model data with its corresponding original CAD design, .stl files were imported in CloudCompare freeware (EDF R\&D, France) for further comparison. A dedicated algorithm enabled fine superimposition of the two models. The "cloud-to-mesh" algorithm was used to compare the produced implant to the respective CAD model; the latter served as reference.

Implants were also examined by SEM (JSM-6500F; JEOL, USA) after applying a gold coating (about 10-nm-thick) using the sputtering coating technique (Q150R ES, Quorum, UK). The porosity on the sample surfaces was quantified from SEM images using ImageJ freeware (National Institutes of Health, USA). Additionally, the minimum (xF,min) and maximum (xF,max) Feret diameter of the micropores, as well as three morphological factors, specifically, the aspect ratio (A.R.), roundness (R) and sphericity $(\mathrm{S})$, were evaluated $(\mathrm{N}=5$ SEM images analyzed per sample; $\mathrm{N}=$ 2 samples per implant architecture tested).

Compression tests up to complete collapse were conducted using an Instron mechanical testing machine (model 3343; capacity $1 \mathrm{kN}$; Instron, Norwood, USA) at a constant speed rate of 20 $\mu \mathrm{m} / \mathrm{min}(\mathrm{N}=5$ samples per implant architecture tested). The reaction force was measured with precision force transducers (Instron 2519-104 series load cells; capacity of $500 \mathrm{~N}$; Instron, Norwood, USA). The total surface of GP and GPRC implants where compression loading was applied was measured, and used to calculate the axial compressive stress as the ratio between the measured reaction force and the measured surface. Strain was computed as the difference between two successive positions and the initial height of the tested implant. From these compression tests, two mechanical properties were evaluated, namely: (i) the effective stiffness (MPa), i.e., the mean slope of the linear portions of each stress-strain curve; and (ii) the ultimate stress (MPa), i.e., the maximum stress borne by the tested implant before rupture.

\section{Animals}

Sixteen 12-15-week-old, female, Lewis rats (Janvier, France) were used. The experimental protocols used in this study received approval by the Ethics Committee on Animal Research of Villemin, Paris, France (APAFIS\#611-20105050408574036), and were carried out in accordance with the European Guidelines for Care and Use of Laboratory Animals (Directive 2010/63/EU).

\section{Surgical procedure}

Prior to anesthesia, buprenorphine $\left(0.1 \mathrm{mg} / \mathrm{kg}\right.$; Buprecare ${ }^{\circledR} 0.3$ $\mathrm{mg} / \mathrm{ml}$; Axience, France) and enrofloxacine $\left(0.05 \mathrm{mg} / \mathrm{kg}\right.$; Baytril ${ }^{\circledR}$ 5\%; Bayer HealthCare, France) per animal were administered subcutaneously as sedative and preventive analgesia and as microbial prophylaxis, respectively. Anesthesia was induced, and maintained, using $2-3 \%$ isoflurane in $100 \%$ oxygen. Each animal was placed in right lateral recumbency. The left limb was clipped and aseptically prepared using $10 \%$ povidone iodine.

The surgical procedure was carried out as previously described [29]. Briefly, after lateral femoral approach, an 8-hole PEEKlocking plate (RatFix Plate; RISystem, Switzerland) was applied on the anterior femoral side and secured using 6 self-tapping locking screws (RatFix ShoulderScrew $0.7 \times 6.5 \mathrm{~mm}$; RISystem, Switzerland) after drilling with a $0.3 \mathrm{~mm}$ drill bit (Drill Bit 0.68 $\mathrm{mm}$, RISystem, Switzerland). A 3-mm-long, mid-diaphyseal ostectomy was performed using a $0.44 \mathrm{~mm}$ Gigli saw $(0.44 \mathrm{~mm}$; RISystem, Switzerland) and a dedicated jig (Drill and Saw Guide; RISystem, Switzerland). Based on preliminary experiments assessing bone repair 8 weeks after ostectomy in 2-, 3-, 4-, and 5mm-long bone defects left empty, the 3 -mm-long bone defect was 
selected as the longest, without it being a critical-sized, bone defect (i.e., able to spontaneously form bone) (Supplementary Fig. 2). The bone defect was filled with either GP or GPRC implants $(\mathrm{N}=$ 8 samples per implant architecture tested). Closure of the fascia, subcutaneous planes and skin were accomplished using 5-0 and 40 glycomer 631 sutures according to standard procedures. While animals were under anesthesia, implant position was determined using non-invasive in vivo X-ray micro-CT as described in Section "Design of hydroxyapatite implants". Post-operative analgesia was provided through a subcutaneous injection of buprenorphine $(0.1$ $\mathrm{mg} / \mathrm{kg}$ ) every $12 \mathrm{~h}$ for 3 consecutive days post-surgery. Throughout the post-operative period, the rats were allowed to weight-bear and were monitored on a daily basis by trained animal-care personnel. Food and water were available ad libitum to the animals.

\section{Micro-computed tomography evaluation}

Bone ingrowth and implant integrity were longitudinally evaluated 4, 6 and 8 weeks after surgery using non-invasive in vivo $\mathrm{X}$-ray micro-CT. Each animal was anesthetized using 2-3\% isoflurane in $50 \%$ oxygen $/ 50 \%$ air and radiographic projections were acquired as described in Section "Design of hydroxyapatite implants". Eight-weeks after implantation, the animals were sacrificed using an overdose of barbiturate (Dolethal®; Vétoquinol, France). Immediately after sacrifice, all left femoral bones were excised, cleaned of overlaying muscle tissue, and fixed in $4 \%$ paraformaldehyde at $4^{\circ} \mathrm{C}$ for 4 days. All specimens $(\mathrm{N}=8$ samples per implant architecture tested) were then imaged again using in vitro X-ray micro-CT (Skyscan 1176; Bruker, Belgium) at the following settings: pixel size $=17.7 \mu \mathrm{m}$; source voltage $=80$ $\mathrm{kV}$; source current $=300 \mu \mathrm{A}$; filter $=$ Copper + Aluminum; rotation step $=0.5^{\circ}$; and exposure time $=100 \mathrm{~ms}$.

The images from both in vivo and in vitro $\mathrm{X}$-ray micro-CT acquisitions were reconstructed using NRecon software (v1.6.8.0; Bruker, Belgium). For qualitative analysis of implant integrity, the presence of lines of fracture in the implant was recorded 4, 6 and 8 weeks after surgery. For quantitative analysis of bone ingrowth after 8 weeks of implantation, CTAn software was used to obtain the volume of newly formed bone from the in vitro X-ray micro$\mathrm{CT}$ images (greyscale threshold for bone $=63$ ). In order to differentiate newly formed bone from the HA ceramic substrate, non-implanted implants were scanned using in vitro X-ray microCT settings as described above and were analyzed to obtain a greyscale threshold (120) for the HA ceramic substrate, which was subtracted from the intensities obtained from the explanted implants, as previously described [30]. Two parameters were evaluated, namely: (i) total bone volume $\left(\mathrm{mm}^{3}\right)$, i.e., bone volume formed in the bone defect, both inside and outside of each implant; and (ii) bone volume inside the implant $\left(\mathrm{mm}^{3}\right)$, i.e., bone volume formed solely inside the gyroid structure of each implant. For quantitative analysis of HA ceramic resorption, CTAn software was used to obtain the volume of HA ceramic from in vitro X-ray micro-CT images obtained before implantation and 8 weeks after implantation (greyscale threshold for HA ceramic $=120$ ).

\section{Histology and histomorphometry}

After X-ray micro-CT imaging, each fixed sample was embedded in methylmethacrylate resin and processed for undecalcified histology according to established techniques [31]. All femoral bones were cut lengthwise and the obtained sections were ground down to a thickness of around $100 \mu \mathrm{m}$, polished, and surface-stained using Stevenel blue and van Gieson picrofuchsin stains. Three central stained sections per implant were then visualized using a digital microscope (VHX-2000; Keyence, USA). For each section, and according to established methods [32], the mineralized newly formed bone area (stained in red) was measured (i) as the total bone area $\left(\mu \mathrm{m}^{2}\right)$, i.e., newly formed bone inside and around each implant; (ii) as the bone area inside each implant $\left(\mu \mathrm{m}^{2}\right)$, i.e., newly formed bone inside the gyroid structure of each implant; and (iii) as a percentage (\%) of bone area in the implant border zones and the implant central zone relative to the bone area inside each implant; for the later parameter, areas adjacent to the proximal and distal bone defect borders corresponding to $50 \%$ of the total implant area were considered the "border zone", and the area in the center of implant corresponding to $50 \%$ of the total implant area was considered the "central zone" (Fig. $7(\mathrm{C})$ ). For each section, area $\left(\mu \mathrm{m}^{2}\right)$ of bone marrow-like tissue inside the gyroid structure of each implant was also evaluated.

For each implant with bone formed inside their gyroid structure, the atomic chemical composition analysis of the newly formed bone was performed on one central resin-embedded section using SEM - EDX spectroscopy equipped with analytical UHR Schottky Emission Scanning Electron Microscope SU-70 (Hitachi, Japan) ( $\mathrm{N}=3$ spots analyzed per sample; $\mathrm{N}=5$ samples per implant architecture tested).

\section{Statistical analysis}

All statistical analyses were performed using the GraphPad Prism Software v6.01 (GraphPad Software, Inc., USA). D'Agostino tests were used to test the normality of the analyzed data (effective stiffness, ultimate stress, total bone volume, bone volume inside implants, total bone area, bone area in the implant border zones and the implant central zone, bone marrow-like tissue area, and calcium/phosphorus ratio). For normally distributed data, t-tests were used to examine the effects of implant architecture. For non-normally distributed data, non-parametric Mann-Whitney U tests were used to examine the effects of implant architecture. For all analyses, differences at $\mathrm{p}<0.05$ were considered statistically significant.

\section{Results}

\section{Implant characterization}

HA implants with an internal gyroid structure were successfully produced (Fig. 1(C)) with a reproducibility and accuracy of $\pm 40 \mu \mathrm{m}$ ( $\underline{\text { Fig. } 2}$ ) and an average deviation of $+1.4 \mu \mathrm{m}$ from the intended $\mathrm{CAD}$ models was determined following a Gaussian distribution displaying full width at half maximum of 85 $\mu \mathrm{m}$. The surface macro-topography was visualized using SEM. Grooves approximately 20- $\mu$ m-deep were observed orientated orthogonally to the implant length (Fig. 3(B)). SEM also revealed the implant microporosity (Fig. 3), which was similar between GP and GPRC implants, and consisted of domino-shaped micropores sub-rounded with a low sphericity (Table 1). The manufacturing process used to obtain the GP and GPRC implants did not affect the HA phase: both FTIR spectra and XRD patterns of the grounded GP and GPRC implants exhibited IR bands and diffraction peaks of the initial HA powder (Fig. 4). No other crystalline or amorphous phase was detected.

\section{In vitro and in vivo implant mechanical resistance}

In vitro, GP and GPRC implants exhibited similar mechanical behaviors: both implant architectures tested had a linear response, characterized, however, by different effective stiffnesses: the 
effective stiffness of GPRC implants was $46 \%$ higher than that of GP implants (Table 2). For the GP implants, sudden drops in mechanical stress were visible throughout the compression test of the implants (Supplementary Fig. 3): they corresponded to the collapse of some of the beams within the gyroid structure, and the collapse depended on the beam orientation and on the presence of material defects making the beam more prone to rupture. For the majority of the implants, the appearance of collapsed beams in the structure led to a decrease in the effective stiffness of the sample. The collapse of beams was also observed in GPRC implants, although to a lesser degree, due to the presence of the cortical-like outer shell (Supplementary Fig. 3). Therefore, the presence of the cortical-like outer shell limited the effect of stiffness reduction. Finally, all samples exhibited a brittle rupture mode. For GP implants, rupture occurred when the last beams within the gyroid structure eventually collapsed, while, for GPRC implants, rupture happened when the cortical-like outer shell reached its ultimate stress. GPRC implants exhibited an ultimate stress of about five times higher than that of GP implants (Table 2). The high standard deviations observed for the effective stiffness and for the ultimate stress are due to the imperfect flatness and parallelism of the implant surfaces (proximal and distal).

The GP and GPRC implants tested in the present study were successfully implanted intact in 3-mm-long, segmental, femoral defect in rats (Fig. 5(A)). The one GP implant, which was fractured upon insertion in the bone defect, was replaced. All animals tolerated well the surgical procedure, and all of them bore weight on the operated limb within a day after surgery. Neither inflammation at the surgical site nor rupture of osteosynthesis material was observed in the animals tested. Upon explantation and subsequent histological analysis, neither inflammation nor foreign body reaction were observed in and around the implants.

Integrity of GPRC implants implanted in a load-bearing bone site was improved compared to that exhibited by the GP implants. At least one line of fracture was observed using in vivo X-ray micro-CT in every one of the 8 GP implants, but in only 2 out of 8 GPRC implants 4 weeks post-implantation; similar observations were made after 6 weeks of implantation (data not shown). At the time of explantation (i.e., 8 weeks post-implantation), high resolution in vitro X-ray micro-CT confirmed that all GP implants had multiple fracture lines; 5 out of 8 GP implants displayed areas of completely morselized implant material (Fig. 5(B)) . On the other hand, 2 out of 8 GRPC implants displayed major fragmentation in the outer zone of the implants (Fig. 5(B)).

\section{In vivo bone formation}

In vivo $\mathrm{X}$-ray micro-CT analyses revealed bone ingrowth after 4 weeks of implantation which progressed until the terminal time point of analysis (i.e., 8 weeks post-implantation). Bone was formed inside and around the implants of both architectures tested; bone, however, was preferentially formed in zones adjacent to the bone defect borders of the GP implants but in the core of the implant for the GPRC implants (Fig. 5(B)). Bone formation was not systematically observed within the gyroid structure (specifically, 3 out of 8 GP implants and 2 out of 8 GPRC implants). In vitro $\mathrm{X}$-ray micro-CT analyses showed no differences in either total bone volume or bone volume inside the GP and the GPRC implants after 8 weeks of implantation (Fig. 6(A)). Total bone volume for both implant architectures tested was similar to the total bone volumes measured in 3-mm-long bone defects left empty (Supplementary Fig. 2(B)). In vitro X-ray micro-CT analyses also showed no significant differences in HA ceramic volume before implantation (T0) and after 8 weeks of implantation (T8) (GP implants: $5.63 \pm 0.19 \mathrm{~mm}^{3}$ at T0 vs. $5.81 \pm 0.19 \mathrm{~mm}^{3}$ at T8; GPRC implants: $10.89 \pm 0.76 \mathrm{~mm}^{3}$ at T0 vs. $10.70 \pm 0.87 \mathrm{~mm}^{3}$ at T8). Similarly to X-ray micro-CT analyses, no differences in total bone area or bone area inside each implant were observed using histomorphometric analyses (Fig. 6(B)).

Histological analyses showed that 5 out of 5 GP implants with bone formed inside the gyroid structure displayed continuity between the bone defect borders and bone formed inside these implants (Fig. 7(A)). Conversely, only 1 out of 6 GPRC implants with bone formed inside the gyroid structure displayed such continuity between bone defect borders and newly formed bone. The interfaces between bone defect borders and newly formed bone in the 5 other GPRC implants were connective tissue, with two interfaces also showing some formation of cartilaginous tissue (Fig. 7(A)). The new bone formed inside the GP and GPRC implants, either in continuity to bone defect borders or not, was highly cellularized and displayed bone marrow-like elements containing numerous adipocytes (Fig. 7(B)). Lining osteoblasts were also present in most of the GP implants and in some of the GPRC implants. Blood vessels as well as multinucleated cells were observed inside both implant architectures tested. Although multinucleated cells remained scarce in all implants, they were more frequent in the GP implants than in the GPRC implants and were mainly observed in direct contact with HA ceramic around implant fragments. Small inlets of soft, cartilaginous callus were also observed inside the gyroid structure, but only in the GP implants (5 out of $8 \mathrm{GP}$ implants), and with a surface median of $4865 \mu \mathrm{m}^{2}$. Histomorphometric analyses confirmed the difference of newly formed bone distribution between the GP and GPRC implants, specifically, $78.6 \pm 21.5 \%$ of new bone formed in the border zones in the GP implants and $68.4 \pm 21.1 \%$ of new bone formed in the central zone for the GPRC implants (Fig. 7(C)).

SEM-EDX spectroscopy images revealed newly formed, mineralized bone with osteocyte lacunae and in direct contact with HA ceramic for both implant architectures tested (Fig. 8(A)). Different levels of bone mineralization, corresponding to different electron density (grey levels), were also observed (Fig. 8(A) and (B)); on average, the calcium and phosphorus content, as well as their ratio, of the newly formed bone was similar between both implant architectures tested (Fig. 8(C)) and comparable to values measured for mature cortical bone in the contralateral femurs $(\mathrm{Ca}=21.6 \pm 0.99 \mathrm{wt} \% ; \mathrm{P}=13.43 \pm 0.42 \mathrm{wt} \% ; \mathrm{Ca} / \mathrm{P}=1.61 \pm$ $0.03)$. As another marker for newly formed bone maturation, area of bone marrow-like elements inside the gyroid structure was assessed and was found to be similar between the GP and GPRC implants (Fig. 8(D)).

\section{Discussion}

TPMS-based structures are of special interest for bone repair, but, up to now, lack of proper AM processes has hindered their application with $\mathrm{CaP}$ bioceramics, one of the synthetic materials most used as orthopedic alloplasts. Using a technique based on the impregnation of wax molds, custom-made femoral implants were designed from X-ray micro-CT imaging and produced with an internal gyroid structure. This is the first time that such complex implants are manufactured in $\mathrm{CaP}$ with high accuracy, at levels comparable to those already reported by the authors for gyroidbased cylinders [25] and spheres [28]. To the best of authors' knowledge, the capability and the flexibility of the developed process is currently unmatched and provided technical 
advancement allowing, for the first time, the use of $\mathrm{CaP}$ bioceramics with architecture biomimicking human trabecular and cortical structures in a bone load-bearing environment (e.g., more than $60 \%$ of 3 -node connectivity [33]). In addition to the tailored macro-TPMS structure, the parameters used for the production of the implants of interest to the present study were chosen to enhance the biological response. The surface topologic microstructure of the produced implants (grooves of approximately $20 \mu \mathrm{m}$ deep, resulting from the selected layer thickness during mold 3D-printing) was, indeed, chosen because it had been shown important in cell adhesion, proliferation, and differentiation by providing a hospitable environment for protein adsorption and cell functions $[34,35]$. The solid content of the slurry and the sintering temperature were chosen to induce sub-micropores, whose dimensions are known to have significant effects on osseointegration dynamics and bone formation [36], [37], [38]. In terms of bioinspired materials, the mechanical properties (Table 2) of both the GP and GPRC implants tested in the present study matched the mechanical properties of femoral, trabecular bone (i.e., an effective stiffness of $441 \pm 271 \mathrm{MPa}$ and a rupture compressive strength of $6.8 \pm 4.8 \mathrm{MPa}$ [39]).

Since in vivo biodegradation of sintered HA is very limited [40], the numerous lines of fracture observed in the GP implants after only 4 weeks of implantation (8 out of 8 implants; data not shown) are probably not related to the chemical degradation of the ceramic material; but, instead, to the mechanical stresses experienced during implantation. These fractures resulting in "freemoving" ceramic fragments can explain the presence of cartilage inlets inside almost all of the GP implants tested and at the interface of the two broken GPRC implants with the bone defect segments (Fig. 7). Mechanical instability during bone repair is, indeed, known to lead to higher cartilage formation in the bone callus [41] and may hinder or delay bone fracture healing [42,43]. The aforementioned excessive mechanical stress experienced by the implants in the present study may be due to unsuitable fixation. Optimization of custom-made fixation systems to stabilize bone defect in load-bearing sites, in order to prevent implant fractures and avoid stress shielding phenomena, has been pertinent research focus for many years, and includes investigation of both new materials and innovative topological designs [44,45]. Current manufacturing limitations and the associated costs of such custommade fixation systems, however, have limited their application to small animal models. Another way to mitigate the risk of implant fracture relies on the modification of the implant design: for example, either other TPMS structures with higher elastic moduli but the same porosity and pore size could be used (i.e., based on primitive lattice) [7], or the global implant porosity could be reduced; at the risk, however, to limit its potential use for bone ingrowth (e.g., lower permeability of such structures $[14,16]$ ) applications.

A cortical-like outer shell was chosen as an alternative strategy and used in the present study to improve the mechanical strength of the gyroid structure as evidenced by the increased effective stiffness and the improved implant integrity upon implantation, since only 2 out of 8 GPRC implants exhibited some lines of fracture after 8 weeks of implantation (Fig. 5(B)). This corticallike outer shell did not impede cell and blood vessel infiltration deep inside the respective implants; in fact, bone formed in the core of the GPRC implants tested. This absence of prejudicial effects on bone formation was confirmed by similar bone volume and bone area results between the GP and the GPRC implants tested (Fig. 6). Based on X-ray micro-CT analyses, bone formation was evaluated as $16.5 \pm 2.1 \%$ and $14.7 \pm 1.4 \%$ of implant volume (BV/TV) and filled $27.3 \pm 7.8 \%$ and $40.3 \pm 5.4 \%$ of void space inside the gyroid structure of the GP and GPRC implants, respectively. These percentages of BV/TV and of occupancy of void space in the implants tested in the present study are in the range of values reported for gyroid macroporous implants manufactured in Ti6Al4V (around 12\% of BV/TV) implanted in tibial defects in mini-pigs for 5 weeks [20], and in polytrimethylene carbonate enriched with HA nanoparticles (around $60 \%$ occupancy) implanted in rabbit calvarial defects for 6 weeks [21]. These reported differences in bone formation may be due to the different animal type, bone defect model, and materials (e.g., osteosynthesis material, implant biomaterial) [46] used by aforementioned studies. The cortical-like outer shell neither affected the maturation of the newly formed bone inside the gyroid structure: the $\mathrm{Ca} / \mathrm{P}$ ratios of mineralized, newly formed bone were similar and the areas of bone-marrow like elements were similar for both GP and GPRC implants (Fig. 8).

Although the occupancy of void space $(40.3 \pm 5.4 \%)$ for the GPRC implants is relatively good after 8 weeks of implantation, revised strategies can be envisioned to improve bone formation in these implants. First, the developed AM process allows production of bioceramics with various compositions; in this respect, the slurry preparation and the sintering parameters are steps to adjust in the pertinent methods. Formation and/or recruitment of osteoclasts, for instance, could be promoted. Osteoclasts have been shown, indeed, to closely collaborate with osteoblasts for bone formation and remodeling [47]. Sintered HA, such as used in the present study, are, however, known to lead to low osteoclast recruitment $[40,48]$. In order to improve this recruitment, various ions of biological interest can be, for instance, substituted into the HA crystalline lattice, affecting the intrinsic (e.g., dissolution rate, mechanical properties) and extrinsic (e.g., resorption rate, modulation of cell metabolism) characteristics of the bioceramics. Amongst others, carbonate, silicate, magnesium, strontium, copper and zinc ions alone or in combination are known to modulate the angiogenic and osteogenic potential of HA. More extensive details regarding the effect of ions on the biological response of HA are available in recent reviews $[49,50]$.

Furthermore, bone formation may be improved by changing the TPMS structure. Although TPMS are considered to have untapped potential for bone repair, few in vivo studies have actually assessed their potential in bone repair applications [19], [20], [21]. More complex TPMS-based structures, such as either porosity gradient or a mixed architecture, may help improve subsequent bone ingrowth [51]. Porosity gradients, based on TPMS [52] and other pore architecture features [53,54], have been shown, for example, to promote cell distribution in the center of implants [52,53] and to improve stem cell osteogenic differentiation [54].

One unexpected result observed in the present study was the difference in distribution of the newly formed bone between the GP and the GPRC implants (Fig. 7(C)). Bone was mainly formed in continuity with the bone defect borders in the GP implants, suggesting that distance osteogenesis, by which osteoblasts lay down new bone on the old bone surface [55], is the main phenomenon regarding bone formation in these implants; contact osteogenesis, by which osteoblasts lay down new bone first on the implant surface, is most likely also taking place in these implants. 
In the GPRC implants, new bone was preferentially formed in the implant center, without continuity with the bone defect borders $(5$ out of 6 GPRC implants with bone formed inside the gyroid structure), indicating that contact osteogenesis alone is taking place in this case.

A possible explanation for the observed difference in bone distribution may be that, by adding the cortical-like outer shell in the implants tested in the present study, tissue penetration from the bone defect segments and from the bone marrow cavity was promoted by limiting "competition" from adjacent soft tissues (e.g., surrounding muscle, nerve). Muscle, however, is known to contribute to bone healing by providing important paracrine stimulation to periosteal cells and by contributing some osteoprogenitors involved in bone repair [56,57]. The fact that bone volumes and areas between the two types of implants tested in the present study were similar suggests that other phenomena, helping bone formation in the GPRC implants, may be at play.

Another, most likely, explanation for the difference in the bone distribution may be that signaling molecules and cells involved in bone formation are more concentrated inside the partially closed GPRC implants than in the case of the open GP implants. Bone repair takes place following consecutive, but overlapping, stages of tissue healing which involves hematoma formation, inflammation, granulation-tissue formation, cartilage (soft callus) formation, cartilage mineralization, wowen bone (hard callus) formation, and bone remodeling. By confining the forming hematoma inside the gyroid structure of the GPRC implants, the cortical-like outer shell may locally increase the concentration of proteins, platelets and other blood cells, such as erythrocytes and leukocytes, entrapped within the hematoma. Aggregated platelets and activated leukocytes can further increase the concentration of signaling molecules because they are known to secrete numerous bioactive cytokines and growth factors (e.g., interleukin (IL)1, IL6, IL8, tumor necrosis factor $\alpha$, vascular endothelial growth factor, platelet derived growth factor, transforming growth factor $\beta 1$, basic fibroblast growth factor, insulin-like growth factor 1) [58,59], which are important in the chemotaxis of inflammatory cells, in angiogenesis, and in the migration, proliferation and differentiation of osteoprogenitor cells. Confinement of the hematoma inside the GPRC implants may also modulate the concentrations of molecules (e.g., thrombin, fibrinogen, factor XIII) affecting the fibrin clot architecture and the cross-linking of adhesive proteins (e.g., fibronectin, collagen) to fibrin [58]. All the aforementioned compounds can result in a fibrin network favoring cell adhesion and migration in the GPRC implants more than in the GP implants. Fibrin clot composition, indeed, has already been shown to modulate bone growth in biphasic $\mathrm{CaP}$ granules [60]. Cellular and molecular confinement within the cortical-like outer shell may, thus, induce important changes in the hematoma and promote bone formation deep in the GPRC implants. Nevertheless, such confinement may also affect the other steps of the bone tissue repair process. Undoubtedly, further research is needed in order to elucidate the impact of implant architecture on the various stages of bone healing.

\section{Conclusions}

In the present study, the authors demonstrated, for the first time, that TPMS-based macroporous implants could be produced in HA and successfully implanted in bone load-bearing anatomical sites, as long as a cortical-like outer shell is added to the macroporous gyroid structure. This cortical-like outer shell improved implant mechanical resistance and modified, without impeding, the bone formation process inside the implant gyroid structure. Modulating implant architecture also appeared to be an attractive approach to improve current understanding of cellular and molecular pathways involved in osteogenesis in order to better adapt strategies for the repair of large bone defects. Although production of TPMS-based implants in $\mathrm{CaP}$ is a laborious process, the challenge is matched by the potential of these bioceramics that are well-known biomaterials already proven to be safe in vivo and the most-used bone-related biomaterial in clinics.

\section{Statement of significance}

Architectural features are known to be key parameters for successful bone repair using synthetic bioceramic bone graft. So far, conventional manufacturing techniques, lacking reproducibility and complete control of the implant macroarchitecture, impeded the exploration of complex architectures, such as triply periodic minimal surfaces (TPMS), which are foreseen to have an unrivaled potential for bone repair. Using a new additive manufacturing process, macroporous TPMS-based bioceramics implants were produced in calcium phosphate, characterized and implanted in a femoral defect in rats. The results showed, for the first time, that such macroporous implants can be successfully implanted in anatomical load-bearing sites when a cortical-like outer shell is added. This outer shell also concentrated new bone formation in the implant center, without affecting new bone quantity or maturity.

\section{Acknowledgments}

The authors would like to thank Prof R. Bizios and Dr G. Salazar-Noratto for reading the article and F. Pillier (UPR15CNRS-LISE) for the SEM-EDX analyses.This work was funded by the Agence Nationale de la Recherche (ANR OrthoFLase); and the Centre National de la Recherche Scientifique (PEPS Archibone).

\section{Disclosure Statement}

The authors wish to confirm that there are no known conflicts of interest associated with this publication and that there was no significant financial support for this work that could have influenced its outcome.

\section{References}

[1] J.T. Marino, B.H. Ziran, Use of solid and cancellous autologous bone graft for fractures and nonunions, Orthop Clin North Am. 41 (2010) 15-26. doi:10.1016/j.ocl.2009.08.003.

[2] V. Campana, G. Milano, E. Pagano, M. Barba, C. Cicione, G. Salonna, W. Lattanzi, G. Logroscino, Bone substitutes in orthopaedic surgery: from basic science to clinical practice, J Mater Sci Mater Med. 25 (2014) 2445-2461. doi:10.1007/s10856-014-5240-2.

[3] V. Karageorgiou, D. Kaplan, Porosity of 3D biomaterial scaffolds and osteogenesis, Biomaterials. 26 (2005) 5474-5491. doi:10.1016/j.biomaterials.2005.02.002.

[4] A. Magnaudeix, J. Usseglio, M. Lasgorceix, F. Lalloue, C. Damia, J. Brie, P. Pascaud-Mathieu, E. Champion, Quantitative analysis of vascular colonisation and angio-conduction in porous silicon-substituted hydroxyapatite with various pore shapes in a chick chorioallantoic membrane (CAM) model, Acta Biomater. 38 (2016) 1791-1789. doi:10.1016/j.actbio.2016.04.039.

[5] X. Xiao, W. Wang, D. Liu, H. Zhang, P. Gao, L. Geng, Y. Yuan, J. Lu, Z. Wang, The promotion of angiogenesis induced by three- 
dimensional porous beta-tricalcium phosphate scaffold with different interconnection sizes via activation of PI3K/Akt pathways, Sci Rep. 5 (2015) 9409. doi:10.1038/srep09409.

[6] A. Entezari, S.-I. Roohani-Esfahani, Z. Zhang, H. Zreiqat, C.R. Dunstan, Q. Li, Fracture behaviors of ceramic tissue scaffolds for load bearing applications, Sci Rep. 6 (2016) 28816. doi:10.1038/srep28816.

[7] I. Maskery, L. Sturm, A.O. Aremu, A. Panesar, C.B. Williams, C.J. Tuck, R.D. Wildman, I.A. Ashcroft, R.J.M. Hague, Insights into the mechanical properties of several triply periodic minimal surface lattice structures made by polymer additive manufacturing, Polymer. 152 (2018) 62-71. doi:10.1016/j.polymer.2017.11.049.

[8] F.P.W. Melchels, A.M.C. Barradas, C.A. Van Blitterswijk, J. De Boer, J. Feijen, D.W. Grijpma, Effects of the architecture of tissue engineering scaffolds on cell seeding and culturing, Acta Biomater. 6 (2010) 4208-4217. doi:10.1016/j.actbio.2010.06.012.

[9] J.W. Jung, H.-G. Yi, T.-Y. Kang, W.-J. Yong, S. Jin, W.-S. Yun, D.-W. Cho, Evaluation of the effective diffusivity of a freeform fabricated scaffold using computational simulation, J Biomech Eng. 135 (2013) 84501. doi:10.1115/1.4024570.

[10] E. Saito, Y. Liu, F. Migneco, S.J. Hollister, Strut size and surface area effects on long-term in vivo degradation in computer designed poly(L-lactic acid) three-dimensional porous scaffolds, Acta Biomater. 8 (2012) 2568-2577. doi:10.1016/j.actbio.2012.03.028.

[11] A. Di Luca, A. Longoni, G. Criscenti, C. Mota, C. Van Blitterswijk, L. Moroni, Toward mimicking the bone structure: Design of novel hierarchical scaffolds with a tailored radial porosity gradient, Biofabrication. 8 (2016) 045007. doi:10.1088/1758-5090/8/4/045007.

[12] S. Van Bael, Y.C. Chai, S. Truscello, M. Moesen, G. Kerckhofs, H. Van Oosterwyck, J.P. Kruth, J. Schrooten, The effect of pore geometry on the in vitro biological behavior of human periosteum-derived cells seeded on selective laser-melted Ti6Al4V bone scaffolds, Acta Biomater. 8 (2012) 2824-34. doi:10.1016/j.actbio.2012.04.001.

[13] S.C. Kapfer, S.T. Hyde, K. Mecke, C.H. Arns, G.E. SchröderTurk, Minimal surface scaffold designs for tissue engineering, Biomaterials. $\quad 32 \quad$ (2011) 6875-6882. doi:10.1016/j.biomaterials.2011.06.012.

[14] H. Montazerian, E. Davoodi, M. Asadi-Eydivand, J. Kadkhodapour, M. Solati-Hashjin, Porous scaffold internal architecture design based on minimal surfaces: A compromise between permeability and elastic properties, Mater Des. 126 (2017) 98-114. doi:10.1016/j.matdes.2017.04.009.

[15] T. Kajihara, H. Jinnai, H. Watashiba, Y. Nishikawa, M. Ito, M. Takahashi, Surface curvatures of trabecular bone microarchitecture, Bone. 30 (2002) 191-194. doi:10.1016/s8756-3282(01)00672-x.

[16] A.P.G. Castro, T. Pires, J.E. Santos, B.P. Gouveia, P.R. Fernandes, Permeability versus design in TPMS scaffolds, Materials. 12 (2019) 1313. doi:10.3390/ma12081313.

[17] Z. Gorgin Karaji, M. Speirs, S. Dadbakhsh, J.P. Kruth, H. Weinans, A.A. Zadpoor, S.A. Yavari, Additively manufactured and surface biofunctionalized porous nitinol, ACS Appl Mater Interfaces. 9 (2017) 1293-1304. doi:10.1021/acsami.6b14026.

[18] C. Yan, L. Hao, A. Hussein, P. Young, Ti-6Al-4V triply periodic minimal surface structures for bone implants fabricated via selective laser melting, J Mech Behav Biomed Mater. 51 (2015) 61-73. doi:10.1016/j.jmbbm.2015.06.024.

[19] G. Li, L. Wang, W. Pan, F. Yang, W. Jiang, X. Wu, X. Kong, K. Dai, Y. Hao, In vitro and in vivo study of additive manufactured porous Ti6Al4V scaffolds for repairing bone defects, Sci Rep. 6 (2016) 34072. doi:10.1038/srep34072.

[20] L. Li, J. Shi, K. Zhang, L. Yang, F. Yu, L. Zhu, H. Liang, X. Wang, Q. Jiang, Early osteointegration evaluation of porous Ti6Al4V scaffolds designed based on triply periodic minimal surface models, J Orthop Transl. (2019) EPub ahead of print. doi:10.1016/j.jot.2019.03.003.

[21] O. Guillaume, M.A. Geven, C.M. Sprecher, V.A. Stadelmann, D.W. Grijpma, T.T. Tang, L. Qin, Y. Lai, M. Alini, J.D. de Bruijn, H. Yuan, R.G. Richards, D. Eglin, Surface-enrichment with hydroxyapatite nanoparticles in stereolithography-fabricated composite polymer scaffolds promotes bone repair, Acta Biomater. 54 (2017) 386-388. doi:10.1016/j.actbio.2017.03.006.
[22] A. Ronca, L. Ambrosio, D.W. Grijpma, Design of porous threedimensional PDLLA/nano-hap composite scaffolds using stereolithography, J Appl Biomater Funct Mater. 10 (2012) 249-258. doi:10.5301/JABFM.2012.10211.

[23] R. Gauvin, Y.C. Chen, J.W. Lee, P. Soman, P. Zorlutuna, J.W. Nichol, H. Bae, S. Chen, A. Khademhosseini, Microfabrication of complex porous tissue engineering scaffolds using 3D projection stereolithography, Biomaterials. 33 (2012) 3824-3834. doi:10.1016/j.biomaterials.2012.01.048.

[24] Z. Chen, Z. Li, J. Li, C. Liu, C. Lao, Y. Fu, C. Liu, Y. Li, P. Wang, Y. He, 3D printing of ceramics: A review, J Eur Ceram Soc. 39 (2019) 661-687. doi:10.1016/j.jeurceramsoc.2018.11.013.

[25] B. Charbonnier, C. Laurent, G. Blanc, O. Valfort, D. Marchat, Porous bioceramics produced by impregnation of 3D-printed wax mold: Ceramic architectural control and process limitations, Adv Eng Mater. 18 (2016) 1728-1737. doi:10.1002/adem.201600308.

[26] D. Marchat, E. Champion, Ceramic devices for bone regeneration, in: P. Palmero, F. Cambier, E. De Barra (Eds.), Adv Ceram Biomater, Elsevier, 2017: pp. 279-311. doi:10.1016/B978-0-08-1008812.00008-7.

[27] D. Marchat, D. Bernache-Assollant, E. Champion, Cadmium fixation by synthetic hydroxyapatite in aqueous solution-Thermal behaviour, J Hazard Mater. 139 (2007) 453-460. doi:10.1016/j.jhazmat.2006.02.040.

[28] B. Charbonnier, C. Laurent, D. Marchat, Porous hydroxyapatite bioceramics produced by impregnation of 3D-printed wax mold: Slurry feature optimization, J Eur Ceram Soc. 36 (2016) 4269-4279. doi:10.1016/j.jeurceramsoc.2016.06.005.

[29] L. Poser, R. Matthys, P. Schawalder, S. Pearce, M. Alini, S. Zeiter, A standardized critical size defect model in normal and osteoporotic rats to evaluate bone tissue engineered constructs, Biomed Res Int. 2014 (2014) 348635. doi:10.1155/2014/348635

[30] J.L. Simon, S. Michna, J.A. Lewis, E.D. Rekow, V.P. Thompson, J.E. Smay, A. Yampolsky, J.R. Parsons, J.L. Ricci, In vivo bone response to 3D periodic hydroxyapatite scaffolds assembled by direct ink writing, J Biomed Mater Res - Part A. 83 (2007) 747-758. doi:10.1002/jbm.a.31329.

[31] H. Petite, V. Viateau, W. Bensaïd, A. Meunier, C. de Pollak, M. Bourguignon, K. Oudina, L. Sedel, G. Guillemin, Tissue-engineered bone regeneration, Nat Biotechnol. 18 (2000) 959-963. doi:10.1038/79449.

[32] L.-E. Monfoulet, P. Becquart, D. Marchat, K. Vandamme, M. Bourguignon, E. Pacard, V. Viateau, H. Petite, D. Logeart-Avramoglou, The $\mathrm{pH}$ in the microenvironment of human mesenchymal stem cells is a critical factor for optimal osteogenesis in tissue-engineered constructs, Tissue Eng Part A. 20 (2014) 1827-1840. doi:10.1089/ten.tea.2013.0500.

[33] N. Reznikov, H. Chase, Y. Ben Zvi, V. Tarle, M. Singer, V. Brumfeld, R. Shahar, S. Weiner, Inter-trabecular angle: A parameter of trabecular bone architecture in the human proximal femur that reveals underlying topological motifs, Acta Biomater. 44 (2016) 65-72. doi:10.1016/j.actbio.2016.08.040.

[34] N. Gui, W. Xu, D.E. Myers, R. Shukla, H.P. Tang, M. Qian, The effect of ordered and partially ordered surface topography on bone cell responses: A review, Biomater Sci. 6 (2018) 250-264. doi:10.1039/c7bm01016h.

[35] S. Samavedi, A.R. Whittington, A.S. Goldstein, Calcium phosphate ceramics in bone tissue engineering: A review of properties and their influence on cell behavior, Acta Biomater. 9 (2013) 8037-8045. doi:10.1016/j.actbio.2013.06.014.

[36] N. Davison, X. Luo, T. Schoenmaker, V. Everts, H. Yuan, F. Barrère-de Groot, de Bruijn, Submicron-scale surface architecture of tricalcium phosphate directs osteogenesis in vitro and in vivo, Eur Cells Mater. 27 (2014) 281-297. doi:10.22203/eCM.v027a20.

[37] K.A. Hing, B. Annaz, S. Saeed, P.A. Revell, T. Buckland, Microporosity enhances bioactivity of synthetic bone graft substitutes, J Mater Sci Mater Med. 16 (2005) 467-475. doi:10.1007/s10856-005-69881.

[38] K. Zhang, Y. Fan, N. Dunne, X. Li, Effect of microporosity on scaffolds for bone tissue engineering, Regen Biomater. 5 (2018) 115-124. doi:10.1093/rb/rby001. 
[39] X. Wang, S. Xu, S. Zhou, W. Xu, M. Leary, P. Choong, M. Qian, M. Brandt, Y.M. Xie, Topological design and additive manufacturing of porous metals for bone scaffolds and orthopaedic implants: A review, Biomaterials. 83 (2016) 127-141. doi:10.1016/j.biomaterials.2016.01.012.

[40] J. Barralet, M. Akao, H. Aoki, H. Aoki, Dissolution of dense carbonate apatite subcutaneously implanted in Wistar rats, J Biomed Mater Res. $\quad 49$ (2000) 176-182. doi:10.1002/(SICI)10974636(200002)49:2<176::AID-JBM4>3.0.CO;2-8.

[41] T. Miclau, C. Lu, Z. Thompson, P. Choi, C. Puttlitz, R. Marcucio, J.A. Helms, Effects of delayed stabilization on fracture healing, J Orthop Res. 25 (2007) 1552-1558. doi:10.1371/journal.pone.0178059.

[42] P. V. Giannoudis, T.A. Einhorn, D. Marsh, Fracture healing: The diamond concept, Injury. 38 (2007) S3-S6. doi:10.1016/S00201383(08)70003-2.

[43] M.S. Ghiasi, J. Chen, A. Vaziri, E.K. Rodriguez, A. Nazarian, Bone fracture healing in mechanobiological modeling: A review of principles and methods, Bone Reports. 6 (2017) 87-100. doi:10.1016/j.bonr.2017.03.002.

[44] D.J. Hak, R. Banegas, K. Ipaktchi, C. Mauffrey, Evolution of plate design and material composition, Injury. 49 (2018) S8-S11. doi:10.1016/S0020-1383(18)30295-X.

[45] P. Augat, C. von Rüden, Evolution of fracture treatment with bone plates, Injury. 49 (2018) S2-S7. doi:10.1016/S0020-1383(18)302948.

[46] J.A. McGovern, M. Griffin, D.W. Hutmacher, Animal models for bone tissue engineering and modelling disease, Dis Model Mech. 11 (2018) dmm033084. doi:10.1242/dmm.033084.

[47] K. Henriksen, M.A. Karsdal, T. John Martin, Osteoclast-derived coupling factors in bone remodeling, Calcif Tissue Int. 94 (2014) 88-97. doi:10.1007/s00223-013-9741-7.

[48] N. Patel, S.M. Best, W. Bonfield, I.R. Gibson, K.A. Hing, E. Damien, P.A. Revell, A comparative study on the in vivo behavior of hydroxyapatite and silicon substituted hydroxyapatite granules, J Mater Sci Mater Med. 13 (2002) 1199-1206. doi:10.1023/A:1021114710076.

[49] J.T.B. Ratnayake, M. Mucalo, G.J. Dias, Substituted hydroxyapatites for bone regeneration: A review of current trends, $\mathbf{J}$ Biomed Mater Res - Part B Appl Biomater. 105 (2017) 1285-1299. doi:10.1002/jbm.b.33651.

[50] M. Šupová, Substituted hydroxyapatites for biomedical applications: A review, Ceram Int. 41 (2015) 9203-9231. doi:10.1016/j.ceramint.2015.03.316.

[51] S. Vijayavenkataraman, L. Zhang, S. Zhang, J. Ying Hsi Fuh, W. Feng Lu, Triply periodic minimal surfaces sheet scaffolds for tissue engineering applications: an optimization approach toward biomimetic scaffold design, ACS Appl Bio Mater. 1 (2018) 259-269. doi:10.1021/acsabm.8b00052.

[52] F.P.W. Melchels, B. Tonnarelli, A.L. Olivares, I. Martin, D. Lacroix, J. Feijen, D.J. Wendt, D.W. Grijpma, The influence of the scaffold design on the distribution of adhering cells after perfusion cell seeding, Biomaterials. $\quad 32 \quad$ (2011) 2878-2884. doi:10.1016/j.biomaterials.2011.01.023.
[53] J.M. Sobral, S.G. Caridade, R.A. Sousa, J.F. Mano, R.L. Reis, Three-dimensional plotted scaffolds with controlled pore size gradients: Effect of scaffold geometry on mechanical performance and cell seeding efficiency, Acta Biomater. 7 (2011) 1009-1018. doi:10.1016/j.actbio.2010.11.003.

[54] A. Di Luca, B. Ostrowska, I. Lorenzo-Moldero, A. Lepedda, W. Swieszkowski, C. Van Blitterswijk, L. Moroni, Gradients in pore size enhance the osteogenic differentiation of human mesenchymal stromal cells in three-dimensional scaffolds, Sci Rep. 6 (2016) 22898 doi: $10.1038 /$ srep22898.

[55] J.E. Davies, Understanding peri-implant endosseous healing, J $\begin{array}{lllll}\text { Dent } & \text { Educ. } & 67 & \text { (2003) }\end{array}$ http://www.ncbi.nlm.nih.gov/pubmed/12959168.

[56] R. Abou-Khalil, F. Yang, S. Lieu, A. Julien, J. Perry, C. Pereira, F. Relaix, T. Miclau, R. Marcucio, C. Colnot, Role of muscle stem cells during skeletal regeneration, Stem Cells. 33 (2015) 1501-1511. doi:10.1002/stem.1945.

[57] K. Shah, Z. Majeed, J. Jonason, R.J. O'Keefe, The role of muscle in bone repair: the cells, signals, and tissue responses to injury, Curr Osteoporos Rep. 11 (2013) 130-135. doi:10.1038/jid.2014.371.

[58] H.T. Shiu, P.C. Leung, C.H. Ko, The roles of cellular and molecular components of a hematoma at early stage of bone healing, J Tissue Eng Regen Med. 12 (2018) e1911-e1925. doi:10.1002/term.2622.

[59] P. Kolar, K. Schmidt-Bleek, H. Schell, T. Gaber, D. Toben, G. Schmidmaier, C. Perka, F. Buttgereit, G.N. Duda, The early fracture hematoma and its potential role in fracture healing, Tissue Eng Part B Rev. 16 (2010) 427-434. doi:10.1089/ten.teb.2009.0687.

[60] L. Le Guehennec, E. Goyenvalle, E. Aguado, P. Pilet, R. Spaethe, G. Daculsi, Influence of calcium chloride and aprotinin in the in vivo biological performance of a composite combining biphasic calcium phosphate granules and fibrin sealant, J Mater Sci Mater Med. 18 (2007) 1489-1495. doi:10.1007/s10856-006-0086-x.

Corresponding author:

Esther POTIER

Laboratoire B3OA UMR CNRS 7052 INSERM U1271

Faculté de médecine Université de Paris - Site Villemin

10, Avenue de Verdun

75010 Paris

FRANCE

Tel. 0033.1.57.27.85.66

Fax. 0033.1.57.27.85.71

Assist. 0033.1.57.27.85.70

Email: esther.potier@cnrs.fr 


\section{Table 1. Surface microporosity analysis of GP and GPRC implants.}

Amount (surface/surface), dimension and morphology of the micropores of the GP and GPRC implants from SEM image analysis. AR (aspect ratio); R (roundness); $\mathrm{S}$ (sphericity); $\mathrm{xF}, \mathrm{min}$ and $\mathrm{xF}$, max correspond to the shortest and longest Feret diameter, respectively; $\mathrm{SD}=$ standard deviation; $\mathrm{N}=5 \mathrm{SEM}$ images analyzed per sample; $\mathrm{N}=2$ samples per implant architecture tested. No difference was observed on surface microporosity of GP and GPRC implants.

\begin{tabular}{lllll}
\hline Sample & GP & \multicolumn{3}{l}{ GPRC } \\
\hline & Mean value & $S D$ & Mean value & $S D$ \\
\hline Amount s/s (\%) & 6.6 & 1.2 & 6.7 & 0.9 \\
\hline $\mathbf{x F m i n} / \boldsymbol{\mu m}$ & 0.57 & 0.08 & 0.53 & 0.06 \\
\hline $\mathbf{x F m a x} / \boldsymbol{\mu \mathbf { m }}$ & 1.23 & 0.17 & 1.03 & 0.10 \\
\hline $\mathbf{A R}$ & 2.60 & 0.14 & 2.37 & 0.08 \\
\hline $\mathbf{R}$ & 0.49 & 0.02 & 0.50 & 0.02 \\
\hline $\mathbf{S}$ & 0.58 & 0.04 & 0.54 & 0.02 \\
\hline
\end{tabular}

Table 2. Mechanical properties of GP and GPRC implants.

Mean values and standard deviation (SD) of the effective stiffness and the ultimate stress of the GP and GPRC implants. $\mathrm{p}<0.05$ were considered statistically significant.

\begin{tabular}{llllll}
\hline Sample & GP & \multicolumn{5}{l}{ GPRC } & \\
\hline & Mean value & $S D$ & Mean value & $S D$ & $p$ values \\
\hline $\begin{array}{l}\text { Effective stiffness } \\
\text { (MPa) }\end{array}$ & 447.6 & 58.9 & 653.9 & 84.4 & $<0.0001$ \\
\hline $\begin{array}{l}\text { Ultimate stress } \\
(\mathbf{M P a})\end{array}$ & 4.8 & 0.9 & 23.0 & 16.0 & 0.0079 \\
\hline
\end{tabular}


Figure 1. Manufacturing of HA implants with gyroid porosity (GP) and with gyroid porosity reinforced by a cortical-like outer shell (GPRC).

(A) Schematic representation of the 3D printing machine used to manufacture the wax molds with an adjustable layer thickness along the z-axis of the printer $(6,12,19,25 \mu \mathrm{m})$ and the chosen printing orientation (implant length along the $\mathrm{z}$-axis of the printer) ; (B) CAD models and (C) photographs of the GP (left) and GRPC (right) implants.
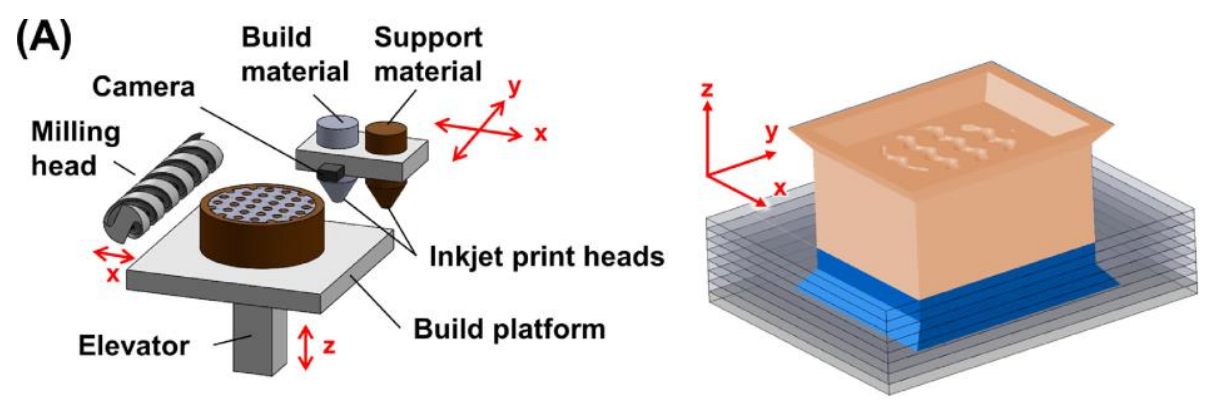

(B)
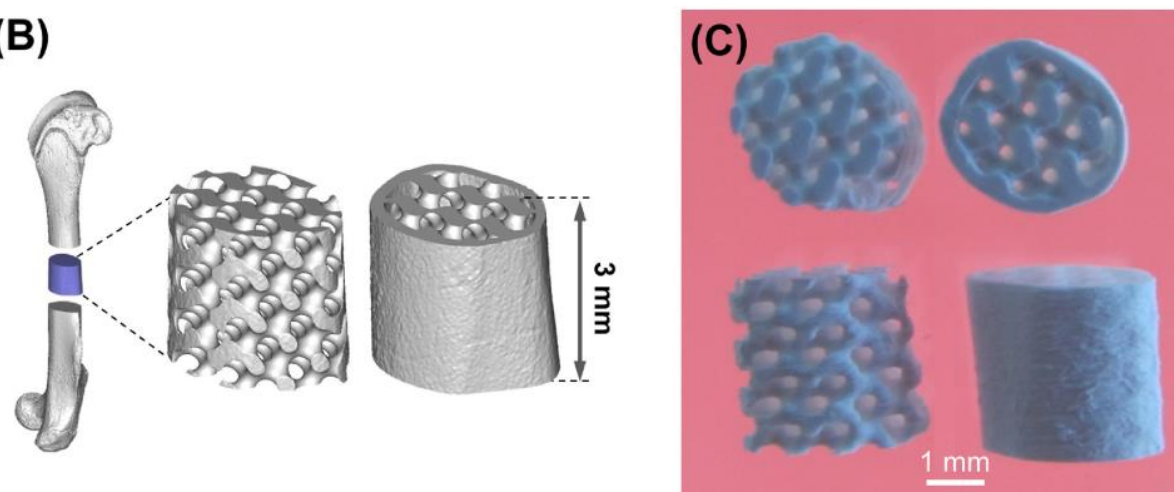


\section{Figure 2. CloudCompare analysis.}

(A) Images show the superposition of the produced GP and GPRC implants obtained using X-ray micro-CT analysis with their respective CAD model. Green indicates good dimensional matching with very little positive or negative deviation; blue and red indicate a lack or an excess of material, respectively. (B) The 3D models of the produced implants displayed excellent matching with their respective CAD models, with a deviation centered on $+1.4 \mu \mathrm{m}$.

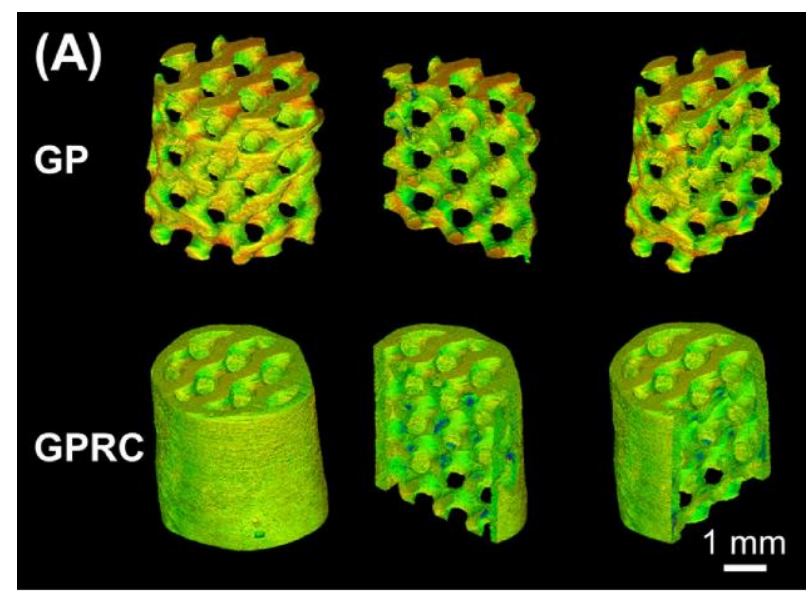

(B)
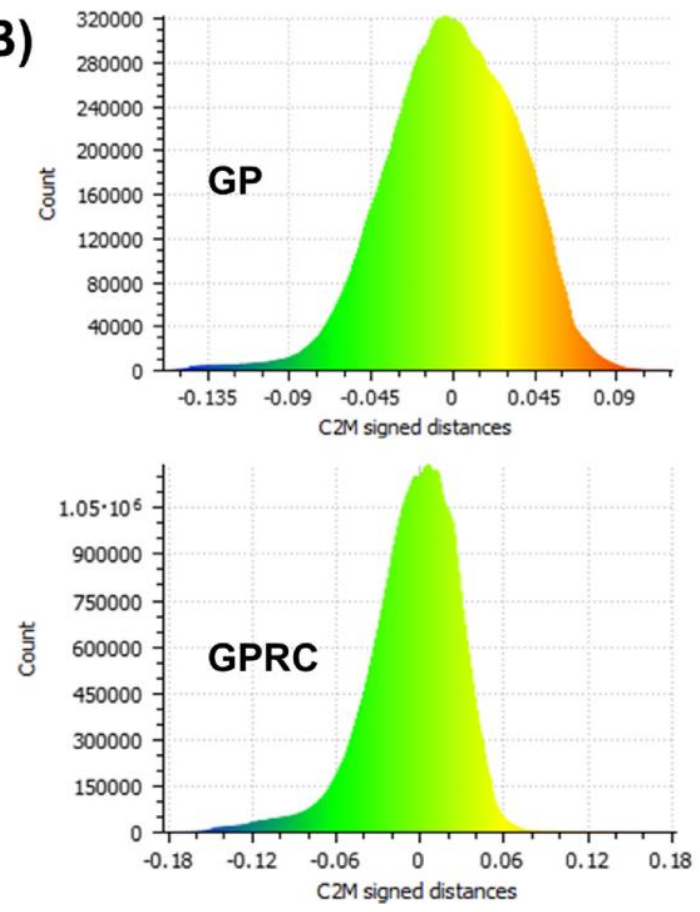


\section{Figure 3. Surface observation of GP and GPRC implants using SEM.}

SEM images of the GP and GPRC implants at different levels of magnification are used to highlight details of their macropore geometry, microtopograhy and micro- to nano-pores. (A) and (B) correspond to the top ((x, y) plan, Fig. 1) and the side ((x or y, z) plan, Fig. 1) views, respectively. Except for the cortical-like outer shell, GP and GPRC implants display the same architectural features.

\section{(A)}
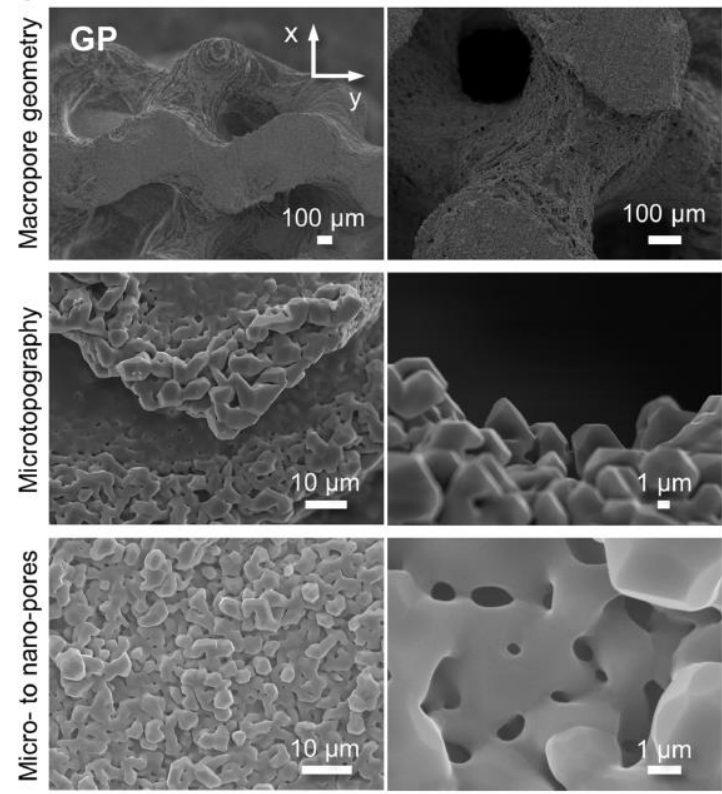

(B)
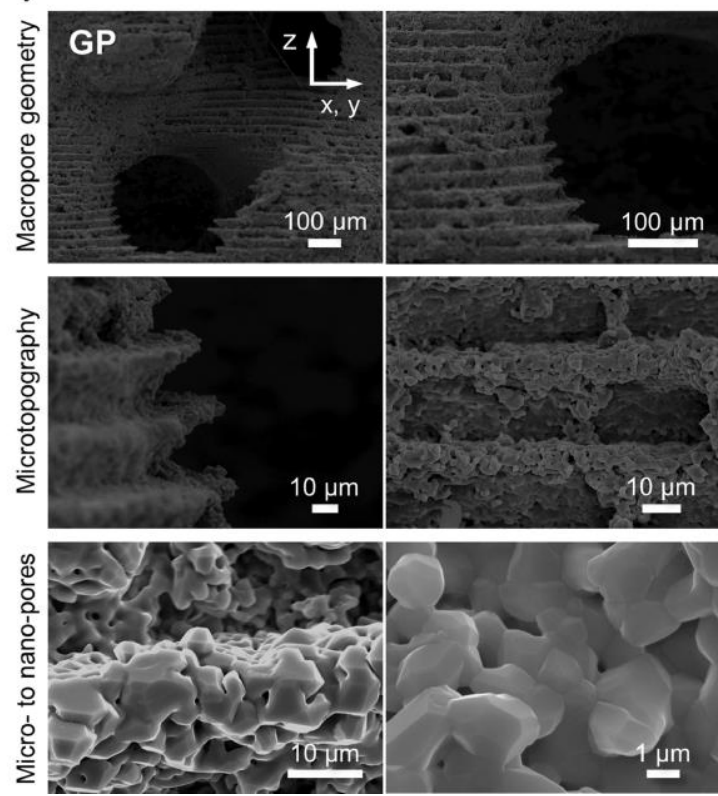
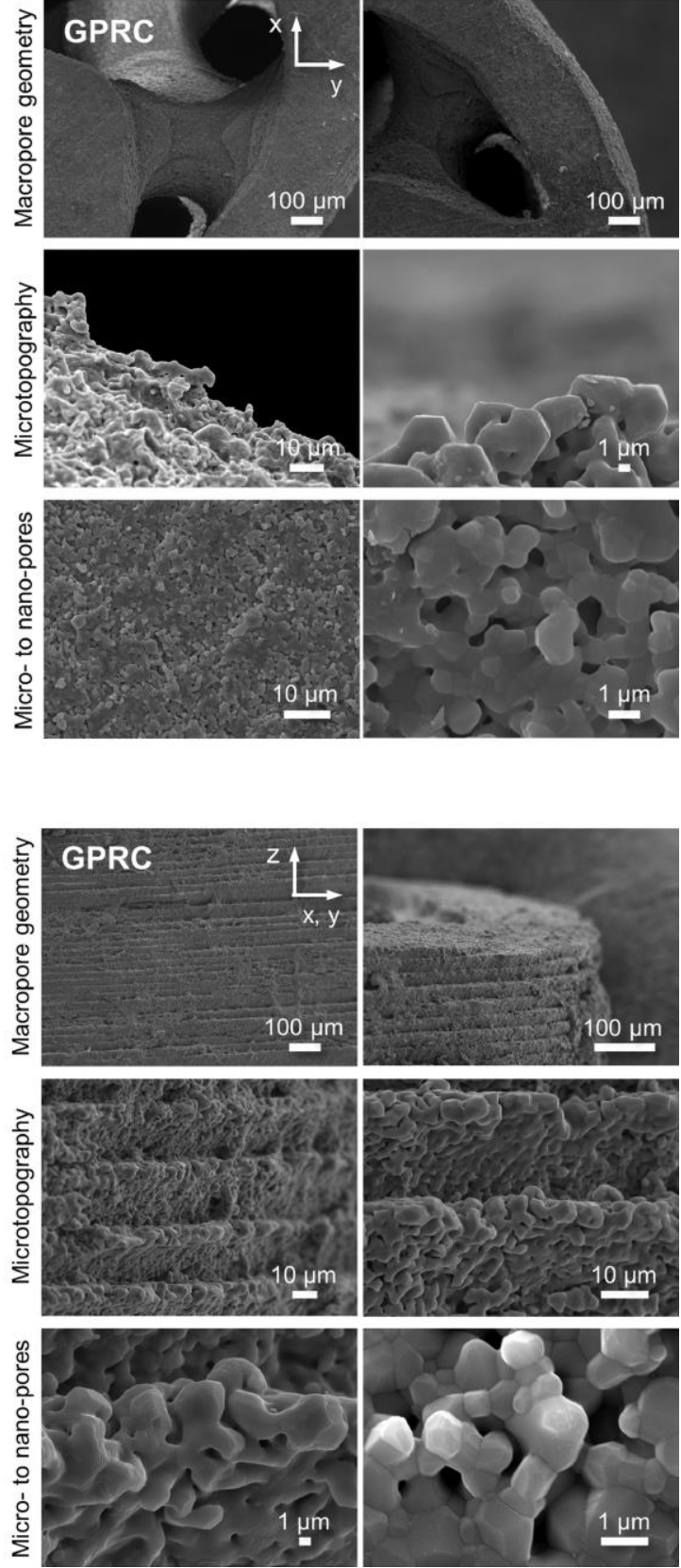
Figure 4. Physico-chemical characterization of HA powder and GP and GPRC implants.

(A) XRD patterns and (B) IR spectra of the HA powder following heat-treatment at $1000^{\circ} \mathrm{C}$ in air for $15 \mathrm{~h}$ and of crushed GP and GPRC implants. ICCPPDF 9-432 was used for XRD phase identification. No difference was observed on XRD patterns and IR spectra between the GP and GPRC implants.
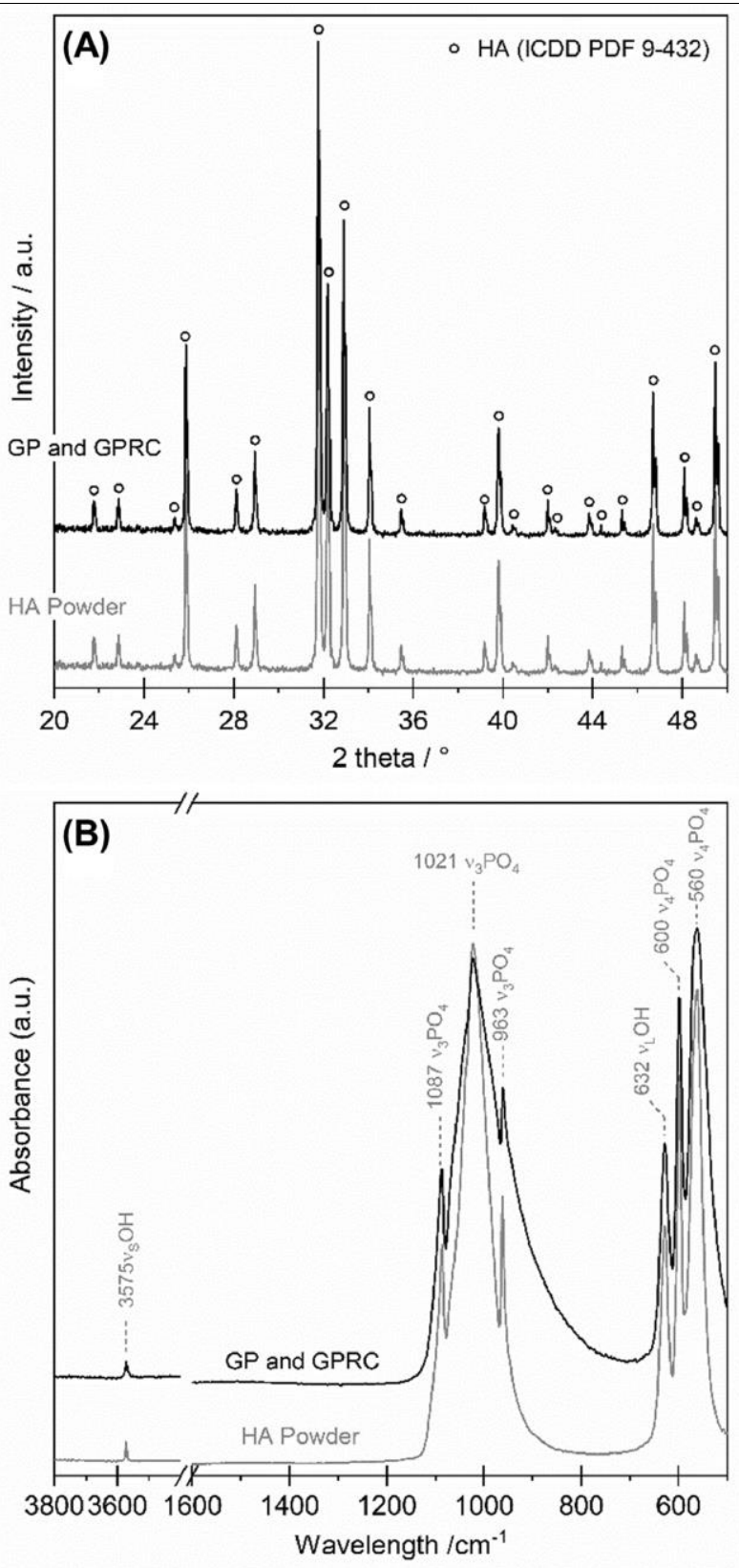
Figure 5. Implant positioning, integrity and bone formation using X-ray micro-CT analyses.

(A) In vivo X-ray micro-CT images of GP and GPRC implants showing the post-implantation positioning of the GP and GPRC implants within a 3-mmlong, femoral defect in Lewis rats. (B) In vitro X-ray micro-CT images of GP and GPRC implants after explantation: 8 out of 8 GP implants and 2 out of 8 GPRC implants displayed at least one line of fracture. Bone was preferentially formed in the border zone of the GP implants but in the central zone of the GPRC implants. Images are representative of $\mathrm{N}=8$ samples per implant architecture tested. White arrow heads indicate zones of fracture.

(A)
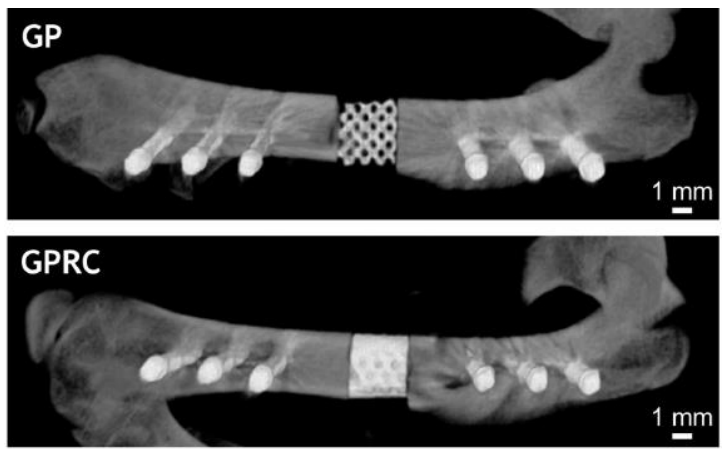

(B)
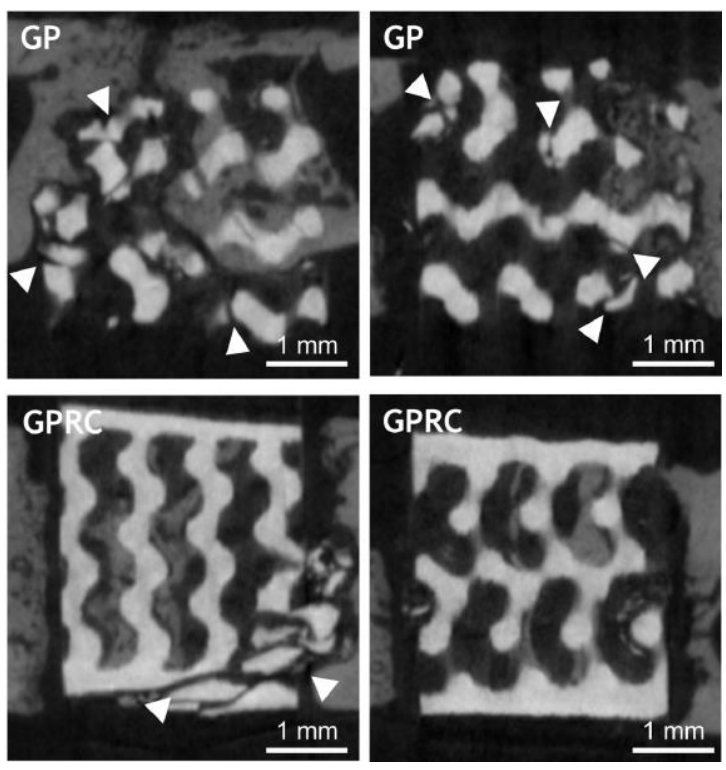


\section{Figure 6. Quantification of newly formed bone.}

(A) The total bone volume and the bone volume inside each implant were determined from X-ray micro-CT images. Values are represented as mean + standard deviation; $\mathrm{N}=8$ samples per implant architecture tested. (B) The total bone area and the bone area inside each implant were determined using histomorphometry. Values are represented as mean + standard deviation; $\mathrm{N}=8$ samples per implant architecture tested. No significant differences were observed on the total bone volume, the bone volume inside each implant, the total bone area, or the bone area inside each implant between the GP and GPRC implants.
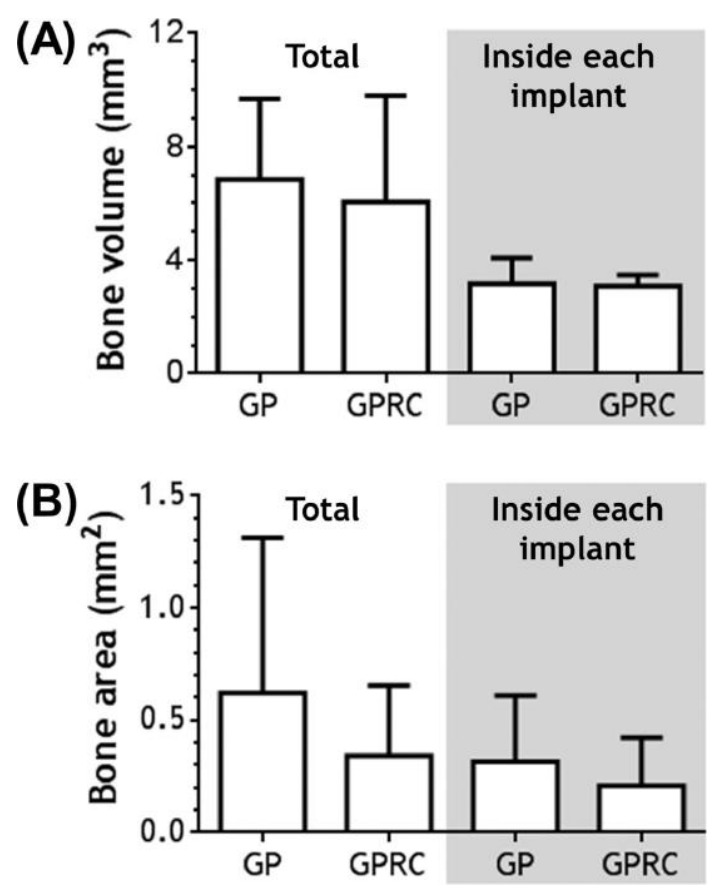


\section{Figure 7. Histological characterization of the tissue formed at the borders and inside the GP and GPRC implants.}

(A) Undecalcified histological sections of the two implant architecture types showing bone formation inside the implants 8 weeks after implantation. Scale bar $=500 \mu \mathrm{m}$. Images are representative of $\mathrm{N}=8$ samples per implant architecture tested. (B) Undecalcified histological sections of the two implants architecture types showing the bone/implant interface at the bone defect margins 8 weeks after implantation. 5 out of 8 GP implants displayed continuity between the borders of the bone defect and the bone formed inside the implant, either on the proximal or distal side. 1 out of 8 GPRC implants displayed such continuity on the proximal side. Scale bar $=20 \mu \mathrm{m}$. Images are representative of $N=8$ samples per implant architecture tested. (C) Undecalcified histological sections of implants showing newly formed, mineralized, and highly cellularized bone tissue inside the gyroid structure, with presence of bone marrow-like elements 8 weeks after implantation in 5 out of 8 GP implants and in 6 out of 8 GPRC implants. Lining osteoblasts could be observed in most of the GP implants and in some of the GPRC implants. Blood vessels and multinucleated cells were observed in both implant architectures. Scale bar $=20$ $\mu \mathrm{m}$. Images are representative of $\mathrm{N}=5$ samples analyzed with bone formed inside the gyroid structure per implant architecture tested. $\mathrm{B}=$ bone at bone defect margins; NB = newly formed bone; Im = implant; CT = connective tissue; Ma = bone marrow-like element; and Cg = cartilaginous tissue. Black arrow heads indicate lining osteoblasts; white arrows indicate multinucleated cells; and empty arrow heads indicate blood vessels. (D) Bone area in the implant border zone and in the implant central zone relative to the bone area inside each implant as determined using histomorphometry. Values are presented as mean + standard deviation; $\mathrm{N}=5$ samples analyzed with bone formed inside the gyroid structure per implant architecture tested. $\mathrm{p}<0.05$ were considered statistically significant.
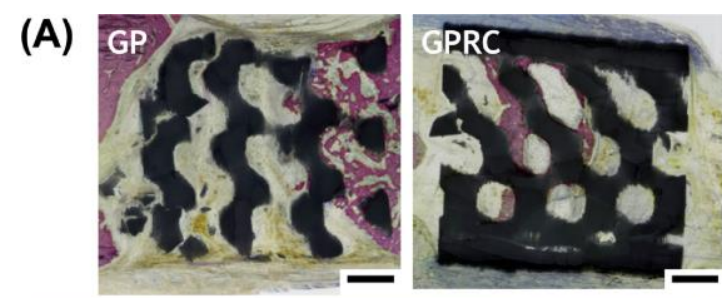

(B)
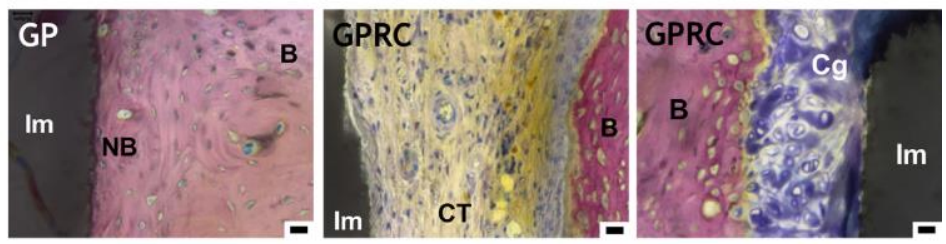

(C)
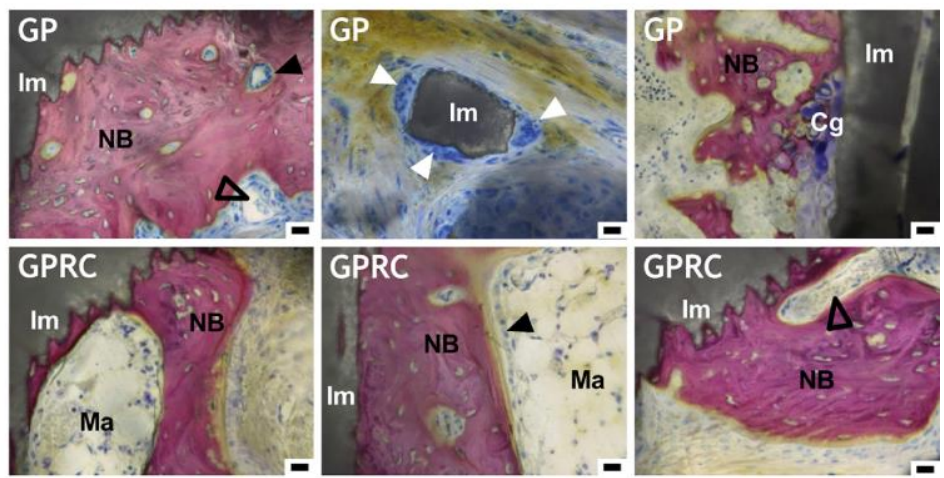

(D)

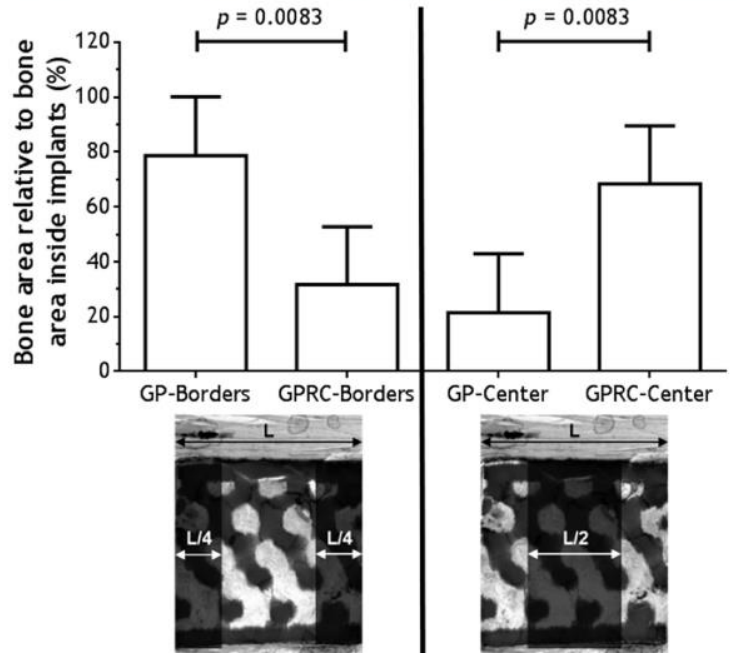




\section{Figure 8. Maturation of newly formed bone inside the GP and GPRC implants.}

(A) SEM images of resin-embedded sections from the central area of GP and GPRC implants (performed in back-scattered electron mode) showing different levels of bone mineralization within the GP and GPRC implants after 8 weeks of implantation. White = HA ceramic; grey = newly formed bone; black = connective or cartilaginous tissue. Images are representative of $\mathrm{N}=5$ samples analyzed with bone formed inside the gyroid structure per implant architecture tested. (B) Magnification of the box region indicated in frame (A) showing disorganized woven bone, with various levels of mineralization; specifically, higher mineralized (lighter grey) woven bone islands surrounded by less mineralized (darker grey) bone areas. (C) Calcium/phosphorus ratio and contents of newly formed bone obtained using SEM-EDX analysis. Values are presented as mean + standard deviation. $\mathrm{N}=5$ samples analyzed with bone formed inside the gyroid structure per implant architecture tested. (D) Bone marrow-like tissue area inside each implant architecture tested, as measured using histomorphometry. Values are presented as mean + standard deviation. $\mathrm{N}=5$ samples analyzed with bone formed inside the gyroid structure per implant architecture tested. No significant differences were observed either on calcium/phosphorus ratio and contents or on bone marrow-like element area between GP and GPRC implants.

(A)

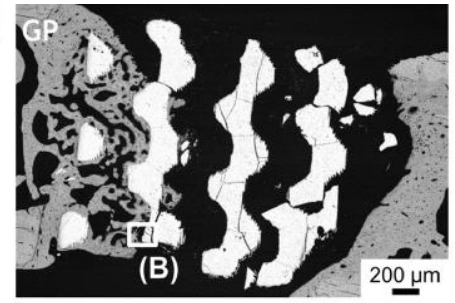

(C)

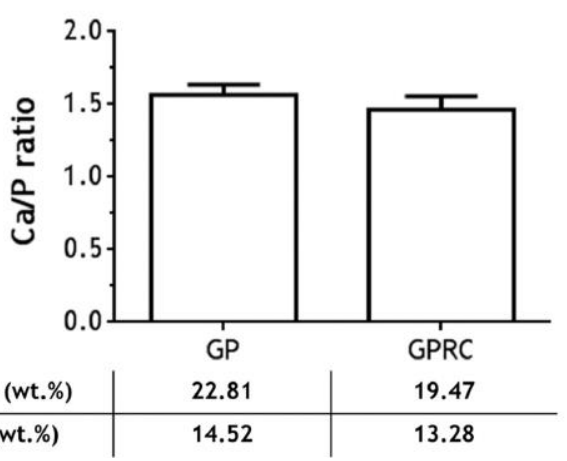

(B)

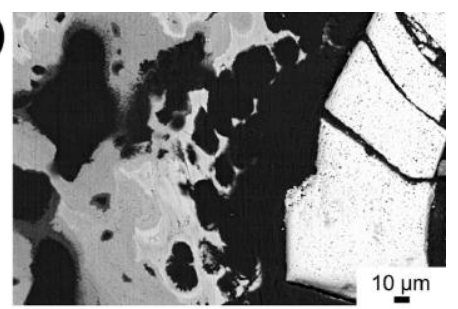

(D)

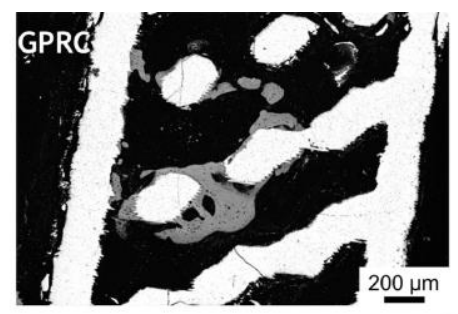

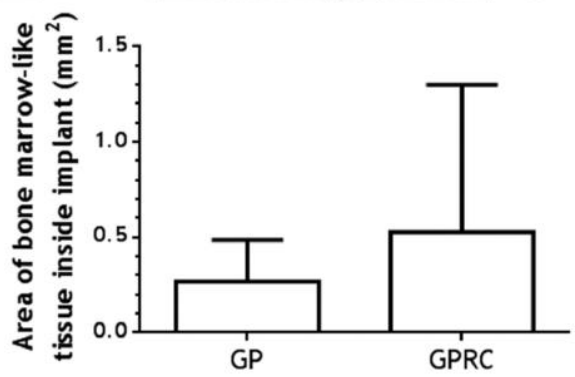




\section{Supplementary Figure 1. Gyroid structure.}

Largest sphere diameter that can move freely through the entire gyroid structure as a function of its porosity and the size of its fundamental unit.

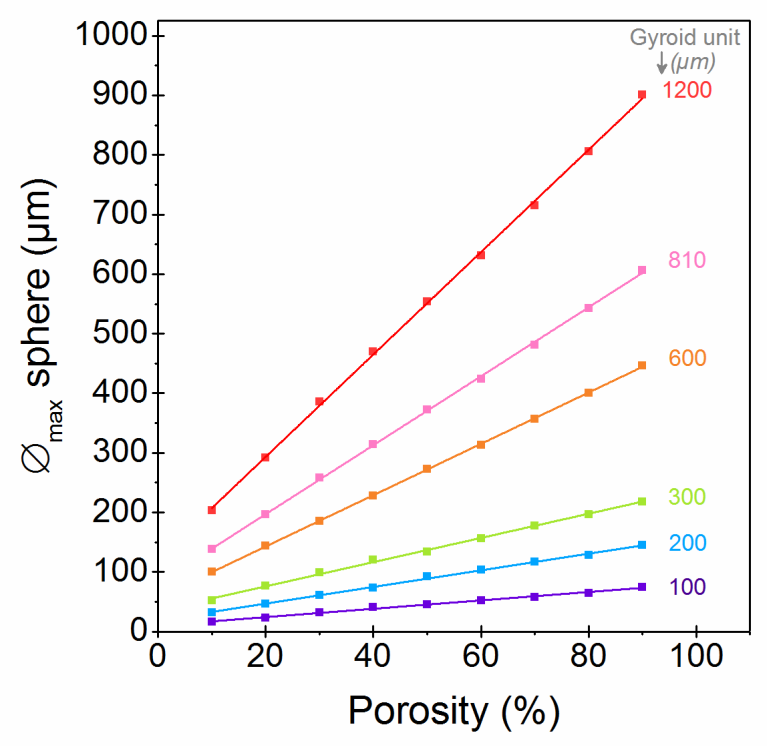




\section{Supplementary Figure 2. Bone formation in bone defects left empty.}

(A) X-ray micro-CT images of 2-, 3-, 4-, and 5-mm-long bone defects in Lewis rats (12 to 15 week-old, females) at the start of the study (T0) and 8 weeks after ostectomy (T8). Images are representative of $\mathrm{N}=3$ animals per bone defect size tested. (B) Bone volume inside bone defects, as determined from $\mathrm{X}$-ray micro-CT images. Values are presented as mean + standard deviation; $\mathrm{N}=3$ animals per bone defect size tested. ND $=$ not detected.
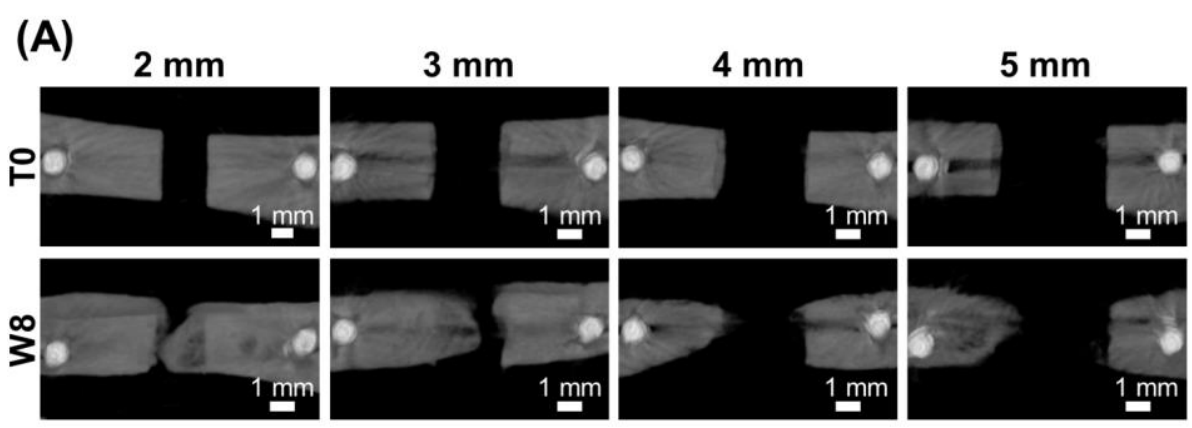

(B)

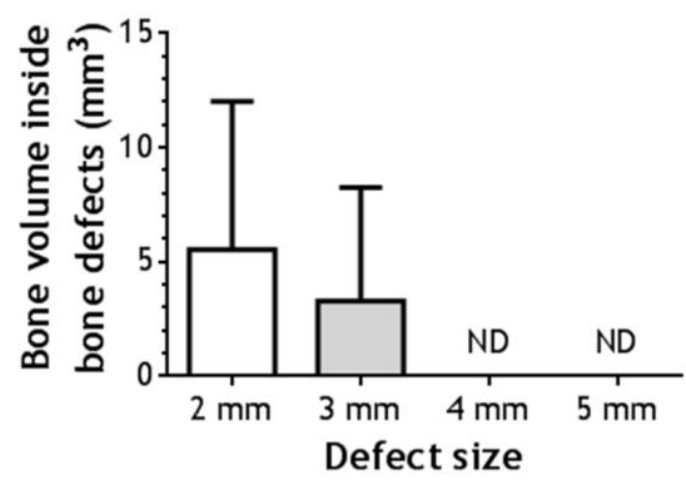




\section{Supplementary Figure 3. Compression load-displacement curves.}

Representative examples of the load-displacements curves of GP and GPRC implants.

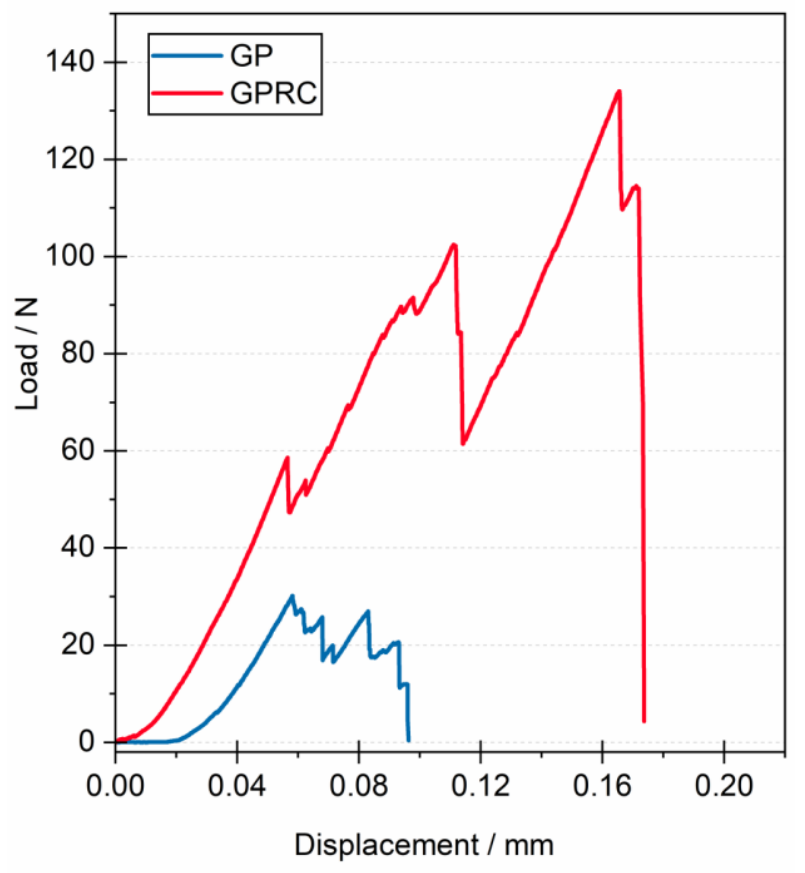

